\title{
Predicting phosphate saturation in silicate magmas: An experimental study of the effects of melt composition and temperature
}

\author{
N. Tollari ${ }^{a, b, *}$, M.J. Toplis ${ }^{\text {b,c }}$, S.-J. Barnes ${ }^{\text {a }}$ \\ ${ }^{a}$ Université du Québec, Chicoutimi, Canada G7H 2 B1 \\ ${ }^{\mathrm{b}} C R P G-C N R S, B P 20, F-54501$, Vandoeuvre-les-Nancy, France \\ ${ }^{\text {c } D T P}$ (UMR 5562), Observatoire Midi-Pyrénées, 14, Av. E. Belin, F-31400 Toulouse, France
}

Received 6 July 2005; accepted in revised form 28 November 2005

\begin{abstract}
A series of 1 atm experiments has been performed to test the influence of iron content and oxidation state on the saturation of phosphate minerals in magmatic systems. Four bulk compositions of different iron content have been studied. The experiments cover a range of temperature from 1030 to $1070{ }^{\circ} \mathrm{C}$ and oxygen fugacity from $1.5 \log$ units below to $1.5 \log$ units above the Fayalite-Magnetite-Quartz buffer. The results demonstrate that neither iron content of the liquid nor oxidation state play a significant role on phosphate saturation. On the other hand, $\mathrm{SiO}_{2}$ and $\mathrm{CaO}$ contents of the liquid strongly influence the appearance of a crystalline phosphate. Our results are combined with data from the literature to define an equation which predicts the $\mathrm{P}_{2} \mathrm{O}_{5}$ content of silicate liquids saturated in either whitlockite or fluorapatite:

$$
M_{\mathrm{P}_{2} \mathrm{O}_{5}}^{\mathrm{liq}-\mathrm{sat}}=\exp \left[T\left(\frac{-0.8579}{139.00-M_{\mathrm{SiO}_{2}}^{\mathrm{liq}}}+0.0165\right)-3.3333 \ln \left(M_{\mathrm{CaO}}^{\mathrm{liq}}\right)\right] \text {, }
$$

where $M$ represents the molar percentage of the relevant oxides and $T$ is temperature in $\mathrm{K}$. This equation is valid over extremely wide ranges of liquid composition and temperature (e.g., $M \mathrm{SiO}_{2}$ from $10 \%$ to $80 \%$ ), including peraluminous liquids. The equation is used to illustrate the relative effects of melt chemistry and temperature on phosphate saturation, both in general terms and in particular for the case of ferrobasaltic differentiation relevant to the late stage differentiation of mafic layered intrusions. It is concluded that magmatic liquids may reach high concentrations in both iron and phosphorus, not through direct association of $\mathrm{P}^{5+}$ and $\mathrm{Fe}^{3+}$, but rather as a consequence of the variations of $\mathrm{CaO}$ and $\mathrm{SiO}_{2}$ content of the liquid. These results may help explain the petrogenesis of certain enigmatic rock types dominated by association of apatite and iron-titanium oxides, such as nelsonites.
\end{abstract}

(c) 2005 Elsevier Inc. All rights reserved.

\section{Introduction}

Apatite is a common mineral in many plutonic rocks, ranging from granites (e.g., Bea et al., 1992) to the late stage cumulates of mafic systems (e.g., Wager and Brown, 1967; Von Gruenewaldt, 1993). Furthermore, volatile-free phosphates, for example whitlockite, are present in igneous rocks of extraterrestrial origin, such as lunar samples (Griffen et al., 1972) and achondrite meteorites (Delaney et al.,

\footnotetext{
${ }^{*}$ Corresponding author.

E-mail address: ntollari@uqac.ca (N. Tollari).
}

1984; Lundberg et al., 1988). The presence of a crystalline phosphate in such rocks is of interest because it provides a potential constraint on the composition of the liquid with which the minerals were in equilibrium or the conditions of temperature and pressure. For example, the work of Watson (1979) and Harrison and Watson (1984) showed that temperature and liquid composition, in particular $\mathrm{SiO}_{2}$ content, are important factors affecting how much $\mathrm{P}_{2} \mathrm{O}_{5}$ is required to saturate silicate melts. The model proposed by Harrison and Watson (1984) has been shown to work well for peralkaline and subaluminous granites, although modification is required when predicting apatite 
saturation in peraluminous compositions (Bea et al., 1992; Pichavant et al., 1992; Wolf and London, 1994). Furthermore, it has been suggested that $\mathrm{SiO}_{2}$ concentration is not the only compositional factor affecting apatite saturation in granitic systems and that $\mathrm{CaO}$ content may also play a role (Bea et al., 1992).

Mafic systems have received less attention than felsic systems, a notable exception being the study of Sha (2000) who considered both apatite and whitlockite saturation in a wide range of liquid compositions at temperatures in the range $1200-1400{ }^{\circ} \mathrm{C}$. However, the fact remains that few experimental data exist for phosphate saturation in ferrobasaltic liquids at the temperatures corresponding to the late stage differentiation of layered intrusions such as the Skaergaard (e.g., Wager, 1960) or the Bushveld (Harney and Von Gruenewaldt, 1995) and it is with these systems in mind that the present study was initiated.

One potentially important characteristic of evolved mafic systems is the high $\mathrm{FeO}^{*}$ content of the liquids (total iron expressed as $\mathrm{FeO}$ ) which may reach $\sim 18 \mathrm{wt} \%$ (Brooks et al., 1991; Toplis and Carroll, 1995), or possibly even more (McBirney and Naslund, 1990). Such high iron contents may affect phosphate saturation because of association of $\mathrm{P}^{5+}$ and $\mathrm{Fe}^{3+}$ in the liquid (Mysen, 1992; Gwinn and Hess, 1993; Toplis et al., 1994a,b). For example, the existence of $\mathrm{P}-\mathrm{Fe}^{3+}$ complexes has been held responsible for the fact that small additions of $\mathrm{P}_{2} \mathrm{O}_{5}$ dramatically influence the stability field of magnetite (Toplis et al., 1994a). This is also consistent with the general finding that $\mathrm{P}^{5+}$ is associated with trivalent cations in silicate liquids, for example, rare earth elements (Ryerson and Hess, 1978), and $\mathrm{Al}^{3+}$ (Toplis and Schaller, 1998; Schaller et al., 1999).

A strong interaction of $\mathrm{P}^{5+}$ and $\mathrm{Fe}^{3+}$ in silicate liquids may therefore retard the saturation of both crystalline phosphates and iron-titanium oxides during magmatic differentiation, leading to extremely high concentrations of $\mathrm{Fe}$ and $\mathrm{P}$ in the liquid. A further consequence of this interaction may be that once either magnetite or apatite finally appears on the liquidus, the other phase will precipitate in abundance. This in turn may potentially explain the petrogenesis of enigmatic rock types dominated by apatite and iron-titanium oxides such as nelsonites found at the highest stratigraphic levels of certain layered intrusions and anorthosites (Philpotts, 1967; Davies and Cawthorn, 1984; Vermark and Von Gruenewaldt, 1986; Cimon, 1998; Dymek and Owens, 2001; Nabil, 2003; Barnes et al., 2004).

In order to test the influence of melt composition on phosphate saturation, in particular the role of ferric iron, we have experimentally saturated ferrobasaltic compositions of variable iron content over a wide range of oxygen fugacity $\left(f \mathrm{O}_{2}\right)$ at $1 \mathrm{~atm}$. Our new data are combined with the literature database of liquid compositions coexisting with crystalline phosphate to construct a predictive model for saturation of apatite $\left(\mathrm{Ca}_{5}\left(\mathrm{PO}_{4}\right)_{3}(\mathrm{OH}, \mathrm{F}, \mathrm{Cl})\right)$ and whitlockite $\left(\left(\mathrm{Ca}, \mathrm{Mg}, \mathrm{Fe}^{2+}\right)_{3}\left(\mathrm{PO}_{4}\right)_{2}\right)$ valid over extremely wide ranges of liquid composition. Some applications to natural systems are briefly discussed.

\section{Experimental approach and methods}

\subsection{Starting materials and compositions studied}

The starting materials used for these experiments were synthetic glass powders prepared from mixtures of reagent grade oxides $\left(\mathrm{SiO}_{2}, \mathrm{TiO}_{2}, \mathrm{Al}_{2} \mathrm{O}_{3}, \mathrm{Fe}_{2} \mathrm{O}_{3}\right.$ and $\left.\mathrm{MgO}\right)$ and carbonates $\left(\mathrm{CaCO}_{3}, \mathrm{Na}_{2} \mathrm{CO}_{3}\right.$ and $\left.\mathrm{K}_{2} \mathrm{CO}_{3}\right)$. Two different $\mathrm{P}$-free compositions were prepared: $\mathrm{SC} 4$, the ferrobasaltic composition studied by Toplis et al. (1994a) which contains $\sim 15 \mathrm{wt} \%$ $\mathrm{FeO}^{*}$, and SC4-8, a composition which maintains the same relative proportions of all oxides as $\mathrm{SC} 4$ with the exception of $\mathrm{FeO}^{*}$, which is present at a level of only $\sim 8 \mathrm{wt} \%$ (Table 1). These compositions, representative of natural liquids, were chosen because the phase relations of SC4 are known as a function of temperature and oxygen fugacity (Toplis and Carroll, 1995) and because the effects of adding $\mathrm{P}_{2} \mathrm{O}_{5}$ have been quantified at $1072{ }^{\circ} \mathrm{C}$ (Toplis et al., 1994a).

Two different amounts of $\mathrm{P}_{2} \mathrm{O}_{5}(5$ and $10 \mathrm{wt} \%)$ were added to each base composition to ensure saturation in a crystalline phosphate. Glasses SC4(5) and SC4(10) (where the number in parentheses is the $\mathrm{P}_{2} \mathrm{O}_{5}$ content) are those whose density was measured by Toplis et al. (1994b), while glasses SC4-8(5) and SC4-8(10) were synthesised for this study. The P-free base compositions were heated above their liquidus in air for $1 \mathrm{~h}$ in thin-walled platinum crucibles. The liquids were quenched by pouring onto a metal plate, then crushed and remelted for another hour to ensure chemical homogeneity. Phosphorous was incorporated as $\mathrm{P}_{2} \mathrm{O}_{5}$ through addition of $\mathrm{NH}_{4} \mathrm{PO}_{3}$ (for composition $\mathrm{SC} 4$ ) and $\mathrm{H}_{3} \mathrm{PO}_{4}$ (for composition SC4-8). The P-bearing compositions were then remelted and crushed two further times. In this way, four bulk compositions have been studied, although it should be appreciated that the liquids present at the end of each experiment are variable in composition because of the different phase relations as a function of temperature and $f \mathrm{O}_{2}$ as detailed below.

\subsection{Experimental techniques}

All experiments were carried out in a vertical rapid quench gas mixing furnace at atmospheric pressure (described by Toplis et al., 1994a). Although working at 1 bar has the drawback that whitlockite rather than apatite crystallises in this volatile-free system, it has the advantage that oxygen fugacity may be carefully controlled and

Table 1

Starting compositions. All concentration in $\mathrm{wt}^{\mathrm{t}} \%$

\begin{tabular}{lcr}
\hline & SC4-B & SC4-8 \\
\hline $\mathrm{SiO}_{2}$ & 49.5 & 54.41 \\
$\mathrm{TiO}_{2}$ & 4.3 & 4.80 \\
$\mathrm{Al}_{2} \mathrm{O}_{3}$ & 11.5 & 12.85 \\
$\mathrm{FeO}^{*}$ & 14.6 & 8.07 \\
$\mathrm{MgO}$ & 4.8 & 5.14 \\
$\mathrm{CaO}$ & 10.0 & 10.84 \\
$\mathrm{Na}_{2} \mathrm{O}$ & 2.9 & 3.35 \\
$\mathrm{~K}_{2} \mathrm{O}$ & 0.48 & 0.54 \\
\hline
\end{tabular}


monitored, an essential consideration when assessing the effect of ferric iron on phosphate saturation. Oxygen fugacity was controlled using $\mathrm{CO}-\mathrm{CO}_{2}$ gas mixtures (Deines et al., 1974) and measured using a yttrium-stabilised zirconia probe. Experiments were carried out at four different $f \mathrm{O}_{2}$ values from $1.5 \log _{10}$ units below the Fayalite-Magnetite-Quartz buffer (FMQ - 1.5) to approximately $1.5 \log _{10}$ units above (FMQ +1.5), as summarised in Table 2 .

For each experiment $\sim 100 \mathrm{mg}$ of starting material was pressed onto a loop of $0.2 \mathrm{~mm}$ diameter platinum wire, using polyvinyl alcohol as a binder. Before each formal experiment the $\mathrm{Pt}$ loops were presaturated in $\mathrm{Fe}$ by equilibration with composition SC4 for $12 \mathrm{~h}$ at $1300{ }^{\circ} \mathrm{C}$ at the relevant $f \mathrm{O}_{2}$ before cleaning in warm HF. A Pt basket, to which four Pt loops were attached (one for each bulk composition), was suspended in the hot spot of the furnace. The furnace temperature was controlled using a Eurotherm 818 controller and measured by an independent $\mathrm{Pt}-\mathrm{Pt}_{10} \mathrm{Rh}$ thermocouple located less than $1 \mathrm{~cm}$ from the samples. Thermocouple calibration was checked against the melting point of gold $\left(1064{ }^{\circ} \mathrm{C}\right)$. The samples were maintained above their liquidus at $1130{ }^{\circ} \mathrm{C}$ for $8 \mathrm{~h}\left(D_{1}\right)$ in order to equilibrate the $\mathrm{Fe}^{3+} / \mathrm{Fe}^{2+}$ ratio of the liquid, then cooled at a constant rate of $3{ }^{\circ} \mathrm{C} / \mathrm{h}$ to the final temperature $\left(1030,1055\right.$ or $\left.1070{ }^{\circ} \mathrm{C}\right)$. This temperature was maintained for a duration $D_{2}$ to allow equilibration between crystalline phases and coexisting melts (see Table 2). All experimental charges were drop-quenched into water.

\subsection{Analytical techniques}

Quenched samples were lightly crushed and mounted as chips in polished sections for petrographic and electron-microprobe analyses. The electron-microprobe analyses were performed using a Cameca SX100 (Université Henri Poincaré, Nancy, France), operated at $15 \mathrm{kV}$ and $15 \mathrm{nA}$ beam

Table 2

Cooling history and run products

\begin{tabular}{|c|c|c|c|c|c|c|c|}
\hline \multirow[t]{2}{*}{ Run no. } & \multicolumn{3}{|c|}{ Cooling history } & \multicolumn{3}{|c|}{ Final conditions } & \multirow{2}{*}{$\begin{array}{l}\text { Run products } \\
\text { Phases }\end{array}$} \\
\hline & $\mathrm{D} 1^{\mathrm{a}}(\mathrm{h})$ & $\operatorname{Ramp}\left({ }^{\circ} \mathrm{C} / \mathrm{h}\right)$ & $\mathrm{D} 2^{\mathrm{b}}(\mathrm{h})$ & $\mathrm{Tf}^{\mathrm{c}}\left({ }^{\circ} \mathrm{C}\right)$ & $\log _{10} f \mathrm{O}_{2}$ & $\Delta \mathrm{FMQ}^{\mathrm{d}}$ & \\
\hline \multicolumn{8}{|l|}{$F M Q-1.5$} \\
\hline $6-\mathrm{sc} 4-\mathrm{b} 5$ & 8 & 3 & 132 & 1055 & -11.795 & -1.51 & Wht, Gl, Pl, Ilm, Cpx \\
\hline 6 -sc4-b10 & 8 & 3 & 132 & 1055 & -11.795 & -1.51 & Wht, Gl, Pl, Mt, Ilm, Qtz, LoCaPx \\
\hline $6-s c 4-85$ & 8 & 3 & 132 & 1055 & -11.795 & -1.51 & Wht, Gl, Pl, Ilm, Psd, Cpx \\
\hline $6-s c 4-810$ & 8 & 3 & 132 & 1055 & -11.795 & -1.51 & Wht, Gl, Pl, Mt, Psd, Qtz, LoCaPx \\
\hline \multicolumn{8}{|l|}{$F M Q-0.5$} \\
\hline 7-sc4-b5 & 8 & 3 & 179 & 1032 & -11.186 & -0.57 & Wht, Gl, Pl, Ilm, Cpx \\
\hline 7-sc4-b10 & 8 & 3 & 179 & 1032 & -11.186 & -0.57 & Wht, Gl, Pl, Mt, Ilm, Qtz, Cpx \\
\hline $7-\operatorname{sc} 4-85$ & 8 & 3 & 179 & 1032 & -11.186 & -0.57 & Wht, Gl, Pl, Mt, Ilm, Qtz, Cpx \\
\hline 7 -sc4-810 & 8 & 3 & 179 & 1032 & -11.186 & -0.57 & Wht, Gl, Pl, Mt, Ilm, Qtz, LoCaPx, Stan \\
\hline $2-\mathrm{sc} 4-\mathrm{b} 5$ & 8 & 3 & 86.5 & 1054 & -10.815 & -0.51 & Wht, Gl \\
\hline 2 -sc4-b10 & 8 & 3 & 86.5 & 1054 & -10.815 & -0.51 & Wht, Gl, Mt, Ilm, Qtz \\
\hline $2-\mathrm{sc} 4-85$ & 8 & 3 & 86.5 & 1054 & -10.815 & -0.51 & Wht, Gl, Pl, Ilm, Psd, Cpx \\
\hline $2-\operatorname{sc} 4-810$ & 8 & 3 & 86.5 & 1054 & -10.815 & -0.51 & Wht, Gl, Pl, Mt, Ilm \\
\hline 3-sc4-b5 & 8 & 3 & 72 & 1075 & -10.472 & -0.47 & Wht, Gl, Pl \\
\hline 3 -sc4-b10 & 8 & 3 & 72 & 1075 & -10.472 & -0.47 & Wht, Gl, Pl, Mt, Qtz \\
\hline $3-\mathrm{sc} 4-85$ & 8 & 3 & 72 & 1075 & -10.472 & -0.47 & Wht, Gl, Pl, Psd, Cpx \\
\hline $3-s c 4-810$ & 8 & 3 & 72 & 1075 & -10.472 & -0.47 & Wht, Gl, Pl, Mt, Ilm, Qtz \\
\hline \multicolumn{8}{|l|}{$F M Q+0.5$} \\
\hline $4-\mathrm{sc} 4-\mathrm{b} 5$ & 8 & 3 & 99 & 1056 & -9.736 & 0.54 & Wht, Gl, Pl, Ilm, Cpx \\
\hline 4-sc4-bl0 & 8 & 3 & 99 & 1056 & -9.736 & 0.54 & Wht, Gl, Pl, Mt, Ilm, Qtz \\
\hline $4-s c 4-85$ & 8 & 3 & 99 & 1056 & -9.36 & 0.54 & Wht, Gl, Pl, Ilm, Qtz, Cpx \\
\hline $4-s c 4-810$ & 8 & 3 & 99 & 1056 & -9.736 & 0.54 & Wht, Gl, Pl, Mt, Ilm, Cpx, Stan \\
\hline \multicolumn{8}{|l|}{$F M Q+1.5$} \\
\hline $5-\mathrm{sc} 4-\mathrm{b} 5$ & 8 & 3 & 82 & 1056 & -8.690 & 1.58 & Wht, Gl, Pl, Mt, Ilm, Qtz, Cpx \\
\hline 5 -sc4-b10 & 8 & 3 & 82 & 1056 & -8.690 & 1.58 & Wht, Gl, Pl, Mt, Ilm, Qtz, Cpx, Stan \\
\hline $5-\operatorname{sc} 4-85$ & 8 & 3 & 82 & 1056 & -8.690 & 1.58 & Wht, Gl, Pl, Mt, Psd, Qtz, Cpx \\
\hline $5-s c 4-810$ & 8 & 3 & 82 & 1056 & -8.690 & 1.58 & Wht, Gl, Pl, Mt, Ilm, Qtz, Cpx, Stan \\
\hline \multicolumn{8}{|l|}{$F M Q+5.5$} \\
\hline 8 -sc4-b5 & 8 & 3 & 140.5 & 1055 & -4.950 & 5.34 & Wht, Gl, Pl, Mt, Ilm, Qtz, Cpx \\
\hline
\end{tabular}

${ }^{a}$ D1:duration above the liquidus to allow the equilibration of the iron redox ratio, before cooling.

${ }^{b}$ D2:duration at the final temperature.

c Tf: final temperature of the experiment.

${ }^{\mathrm{d}} \Delta$ FMQ: represents $\log f \mathrm{O}_{2}$ (experiment) - $\log f \mathrm{O}_{2}$ (FMQ buffer).

e Phases: Wht, whitlockite; Gl, glass; Pl, plagioclase; Cpx, clinopyroxene; LoCaPx, low-Ca pyroxene; Mt, magnetite-ulvöspinel solid solution; Ilm, ilmenite-haematite solid solution; Psd, pseubroockite- $\mathrm{Fe}_{2} \mathrm{TiO}_{5}$ solid solution; Qtz, quartz; Stan, stanfieldite. 
current. Standards used were albite for $\mathrm{Na}$ and $\mathrm{Al}$, orthoclase for $\mathrm{Si}$ and $\mathrm{K}$, hematite for $\mathrm{Fe}$, wollastonite for $\mathrm{Ca}$, olivine for $\mathrm{Mg}, \mathrm{MnTiO}_{3}$ for $\mathrm{Ti}$ and chlorapatite for $\mathrm{P}$. Counting times of $20 \mathrm{~s}$ on the peak and $10 \mathrm{~s}$ on the background were typically employed. Incident-beam diameter was $5 \mu \mathrm{m}$ on glass (where space permitted) to minimise alkali volatility under the electron beam. A focused beam $(1-2 \mu \mathrm{m})$ was used in all other cases.

\subsection{Attainment of equilibrium}

At the studied temperatures experiments on bulk compositions SC4(5) and SC4(10) contain liquid proportions typically in excess of $50 \%$ (Figs. 1a and b), a fact which promotes the rate of solid-liquid reactions. In this respect it may be noted that experiments of similar melt fraction, duration and temperature (Toplis et al., 1994a) were demonstrated to be in equilibrium based upon reversal experiments. Furthermore, in the experiments described here, minerals are generally unzoned (as evidenced from backscattered electron images) and the standard deviations of multiple analyses on glass and phosphate are typically small (Table 3 ). All these lines of evidence point to a good approach to equilibrium for these samples. On the other hand, experiments on bulk compositions SC4-8(5) and SC4-8(10) contained consider- ably less glass, typically on the order of $20 \%$. In these cases, some extent of mineral zoning could be observed in backscattered electron images, particularly for pyroxenes (e.g., Fig. 1c) and the standard deviations of multiple analyses of glasses are greater than for the bulk composition SC4, although those of whitlockite remain small (Table 3). However, although the approach to equilibrium at the sample scale would not appear to be as complete for experiments using SC4-8 we have reason to believe that local equilibria between phosphate and adjacent liquid were reached, as discussed further below.

\section{Results}

\subsection{Phase equilibria}

The observed phase relations are listed in Tables 2 and 3 and illustrated in Fig. 1. All experiments contain whitlockite (Wht) and glass (Gl). In experiments with additions of $10 \mathrm{wt} \% \mathrm{P}_{2} \mathrm{O}_{5}$ (but not in experiments with additions of $5 \mathrm{wt} \% \mathrm{P}_{2} \mathrm{O}_{5}$ ) two coexisting liquids occur, an observation consistent with the fact that $\mathrm{P}$ is known to promote liquid-liquid immiscibility in silicate systems (e.g., Visser and Koster van Groos, 1979). Although the high bulk $\mathrm{P}_{2} \mathrm{O}_{5}$ concentration in these experiments precludes any di-

$\mathrm{b}$

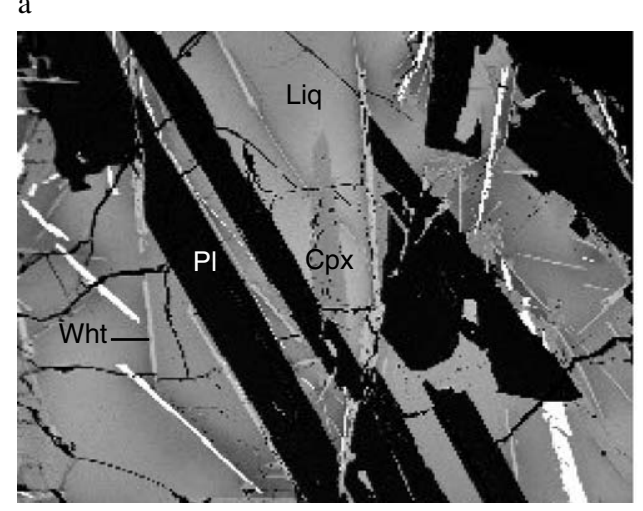

$-50 \mu \mathrm{m}$
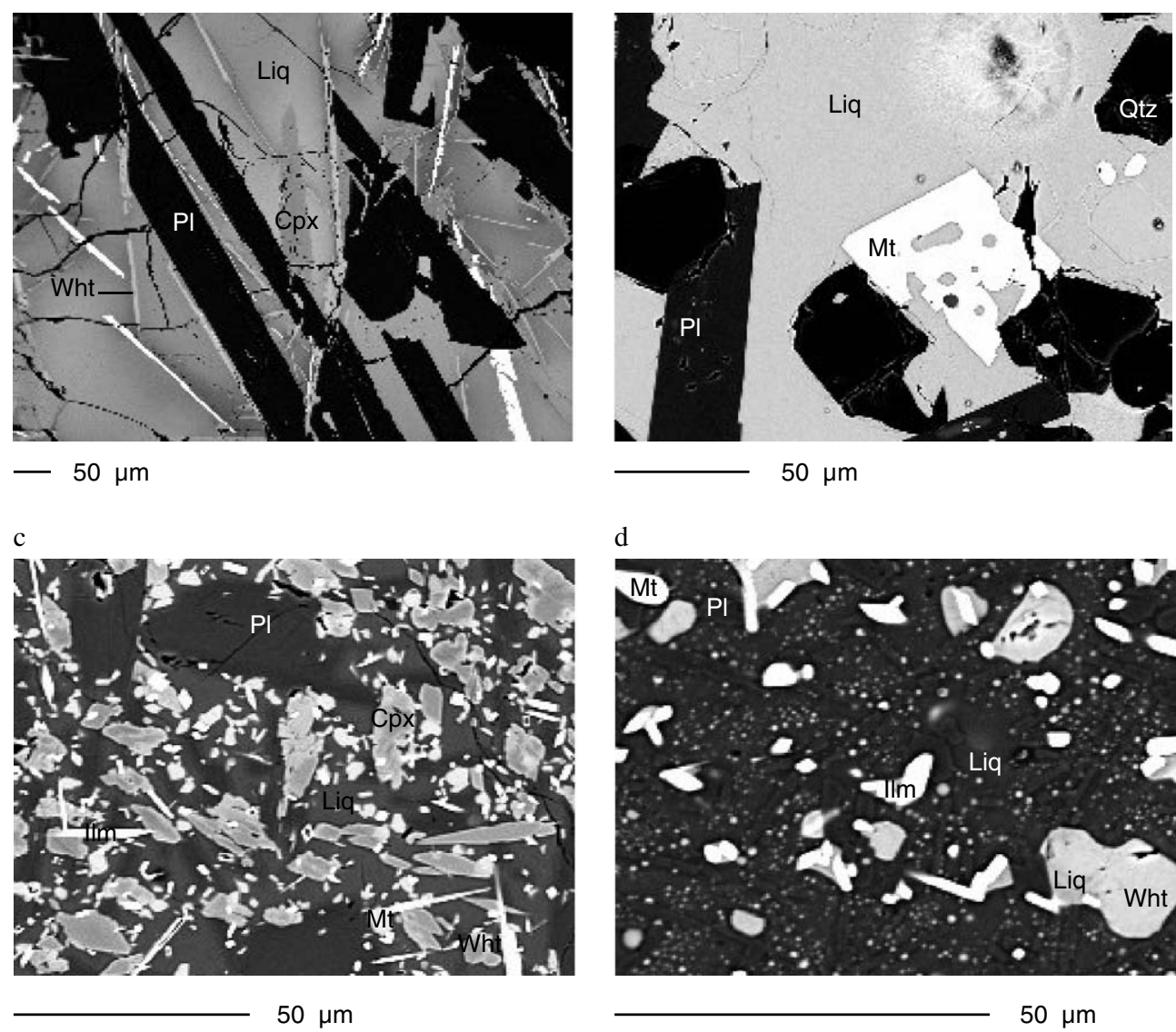

d

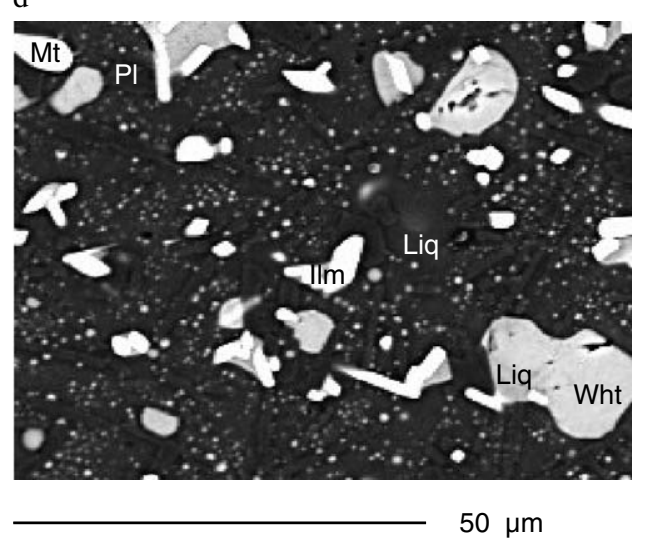

Fig. 1. Backscattered electron images of the FMQ +1.5 run products quenched from $1055^{\circ} \mathrm{C}$. (a) Sample SC4-B-5: Liq $+\mathrm{Wht}+\mathrm{Pl}+\mathrm{Mt}+\mathrm{Ilm}+$ $\mathrm{Qtz}+\mathrm{Cpx}$, (b) sample SC4-B-10: $\mathrm{Liq}+\mathrm{Wht}+\mathrm{Pl}+\mathrm{Mt}+\mathrm{Ilm}+\mathrm{Qtz}+\mathrm{Cpx}+\mathrm{Stan}$, (c) sample SC4-8-5: $\mathrm{Liq}+\mathrm{Wht}+\mathrm{Pl}+\mathrm{Mt}+\mathrm{Psd}+\mathrm{Qtz}+\mathrm{Cpx}$, (d) sample SC4-8-10: Liq + Wht $+\mathrm{Pl}+\mathrm{Mt}+\mathrm{Ilm}+\mathrm{Qtz}+\mathrm{Cpx}+\mathrm{Stan}$. 
Table 3

Electron microprobe analyses ( $\mathrm{wt} \%$ ) of run products

\begin{tabular}{|c|c|c|c|c|c|c|c|c|c|c|c|c|c|}
\hline Run no. & Phase $^{\mathrm{a}}$ & $\%$ modal & $\begin{array}{l}\text { Number } \\
\text { of analyses }\end{array}$ & $\mathrm{SiO}_{2}$ & $\mathrm{TiO}_{2}$ & $\mathrm{Al}_{2} \mathrm{O}_{3}$ & $\mathrm{FeO}$ & $\mathrm{MgO}$ & $\mathrm{CaO}$ & $\mathrm{Na}_{2} \mathrm{O}$ & $\mathrm{K}_{2} \mathrm{O}$ & $\mathrm{P}_{2} \mathrm{O}_{5}$ & Total \\
\hline \multicolumn{14}{|l|}{$F M Q-1.5$} \\
\hline \multirow[t]{5}{*}{ 6-sc4-b5 } & Wht & 5 & 9 & $0.18(0.06)$ & $0.05(0.02)$ & $0.04(0.03)$ & $3.80(0.16)$ & $3.07(0.08)$ & $47.17(0.42)$ & $0.25(0.03)$ & $0.02(0.01)$ & $44.79(0.37)$ & 99.37 \\
\hline & Liq & 60 & 8 & $51.01(1.22)$ & $3.62(0.17)$ & $10.51(0.45)$ & $16.95(0.64)$ & $2.86(0.20)$ & $8.65(0.3)$ & $1.95(0.12)$ & $0.77(0.06)$ & $3.06(0.25)$ & 99.38 \\
\hline & Plag & 20 & 1 & 57.84 & 0.15 & 26.69 & 0.54 & 0.17 & 9.07 & 5.53 & 0.28 & 0.05 & 100.31 \\
\hline & $\mathrm{Ilm}$ & 5 & 1 & 0.09 & 52.37 & 0.19 & 42.52 & 3.35 & 0.23 & 0.03 & 0.01 & 0.01 & 98.79 \\
\hline & $\mathrm{Cpx}$ & 10 & 1 & 50.71 & 1.06 & 1.26 & 21.99 & 17.68 & 6.77 & 0.07 & 0.00 & 0.06 & 99.59 \\
\hline \multirow[t]{8}{*}{ 6-sc4-b10 } & Wht & 8 & 6 & $0.32(0.31)$ & $0.08(0.03)$ & $0.08(0.07)$ & $3.83(0.19)$ & $4.07(0.10)$ & $44.92(0.19)$ & $0.20(0.03)$ & $0.02(0.01)$ & $43.48(0.64)$ & 97.00 \\
\hline & Liq 1 & 25 & 4 & $31.56(1.34)$ & $3.78(0.18)$ & $7.42(0.52)$ & $21.30(0.28)$ & $8.57(0.48)$ & $10.41(0.59)$ & $1.12(0.03)$ & $0.25(0.03)$ & $14.68(0.77)$ & 99.09 \\
\hline & Liq 2 & 25 & 7 & $69.19(0.86)$ & $1.39(0.1)$ & $13.20(1.03)$ & $6.17(0.29)$ & $1.53(0.14)$ & $2.79(0.61)$ & $1.08(0.25)$ & $1.71(0.14)$ & $1.44(0.14)$ & 98.49 \\
\hline & Plag & 20 & 1 & 59.00 & 0.41 & 24.68 & 2.10 & 0.34 & 8.64 & 4.66 & 0.55 & 0.41 & 100.78 \\
\hline & $\mathrm{Mt}$ & 5 & 1 & 0.20 & 16.46 & 3.74 & 71.50 & 3.31 & 0.10 & 0.00 & 0.02 & 0.00 & 95.33 \\
\hline & $\mathrm{Ilm}$ & 3 & 1 & 0.04 & 44.33 & 0.59 & 47.88 & 3.91 & 0.61 & 0.00 & 0.01 & 0.04 & 97.39 \\
\hline & Qtz & 8 & 1 & 97.50 & 0.43 & 2.37 & 0.44 & 0.00 & 0.22 & 0.27 & 0.03 & 0.03 & 101.29 \\
\hline & Lpx & 6 & 1 & 52.19 & 0.65 & 1.65 & 22.83 & 22.64 & 1.05 & 0.03 & 0.00 & 0.12 & 101.17 \\
\hline \multirow[t]{6}{*}{ 6-sc4-85 } & Wht & 22 & 5 & $0.99(0.39)$ & $0.11(0.04)$ & $0.27(0.13)$ & $3.27(0.15)$ & $3.19(0.08)$ & $46.70(0.68)$ & $0.35(0.02)$ & $0.03(0.02)$ & $42.92(0.59)$ & 97.83 \\
\hline & $\mathrm{Liq}$ & 15 & 7 & $72.31(1.77)$ & $2.02(0.14)$ & $11.72(0.28)$ & $4.62(0.82)$ & $0.90(0.07)$ & $3.28(0.51)$ & $0.75(0.21)$ & $1.24(0.05)$ & $0.70(0.37)$ & 97.54 \\
\hline & Plag & 20 & 1 & 57.20 & 0.21 & 26.92 & 0.89 & 0.16 & 10.06 & 5.19 & 0.11 & 0.01 & 100.75 \\
\hline & $\mathrm{Ilm}$ & 5 & 1 & 0.20 & 50.78 & 0.19 & 41.85 & 3.90 & 0.49 & 0.01 & 0.04 & 0.01 & 97.47 \\
\hline & Psd & 8 & 1 & 0.13 & 67.30 & 1.58 & 22.39 & 5.88 & 0.33 & 0.02 & 0.01 & 0.01 & 97.65 \\
\hline & Cpx & 30 & 1 & 50.12 & 1.57 & 2.13 & 17.94 & 17.22 & 10.59 & 0.20 & 0.02 & 1.03 & 100.81 \\
\hline \multirow[t]{7}{*}{$6-s c 4-810$} & Wht & 12 & 8 & $0.43(0.27)$ & $0.09(0.02)$ & $0.10(0.08)$ & $2.96(0.12)$ & $4.78(0.05)$ & $44.55(0.48)$ & $0.23(0.04)$ & $0.03(0.02)$ & $47.01(0.23)$ & 100.16 \\
\hline & Liq & 25 & 6 & $71.73(0.91)$ & $0.95(0.09)$ & $12.96(1.08)$ & $3.21(0.24)$ & $1.12(0.18)$ & $2.27(0.64)$ & $1.20(0.43)$ & $1.35(0.11)$ & $1.63(0.41)$ & 96.42 \\
\hline & Plag & 30 & 1 & 56.07 & 0.17 & 26.87 & 1.34 & 0.16 & 9.67 & 4.87 & 0.15 & 0.27 & 99.57 \\
\hline & $\mathrm{Mt}$ & 10 & 1 & 6.27 & 5.04 & 4.95 & 73.19 & 4.04 & 0.72 & 0.07 & 0.18 & 0.38 & 94.84 \\
\hline & Psd & 5 & 1 & 3.88 & 43.28 & 2.75 & 39.21 & 3.24 & 2.52 & 0.15 & 0.05 & 2.18 & 97.26 \\
\hline & Qtz & 10 & 1 & 91.50 & 0.39 & 5.41 & 0.23 & 0.03 & 0.90 & 0.53 & 0.02 & 0.02 & 99.02 \\
\hline & Lpx & 8 & 8 & $52.10(1.28)$ & $0.59(0.20)$ & $2.95(0.47)$ & $15.17(0.44)$ & $27.49(0.63)$ & $1.14(0.59)$ & $0.04(0.03)$ & $0.03(0.022)$ & $1.00(0.69)$ & 100.510 \\
\hline \multicolumn{14}{|l|}{$F M Q-0.5$} \\
\hline \multirow[t]{5}{*}{7 -sc4-b5 } & Wht & 12 & 7 & $1.42(1.37)$ & $0.13(0.06)$ & $0.31(0.17)$ & $4.33(0.15)$ & $2.64(0.12)$ & $45.39(0.88)$ & $0.26(0.03)$ & $0.07(0.05)$ & $45.15(0.69)$ & 99.70 \\
\hline & Liq & 35 & 6 & $67.20(1.92)$ & $1.43(0.09)$ & $11.46(0.27)$ & $9.21(1.49)$ & $0.77(0.08)$ & $3.98(0.63)$ & $1.24(0.19)$ & $1.99(0.09)$ & $0.70(0.21)$ & 97.98 \\
\hline & Plag & 24 & 1 & 59.08 & 0.18 & 26.29 & 0.64 & 0.14 & 8.82 & 5.34 & 0.25 & 0.06 & 100.81 \\
\hline & $\mathrm{Ilm}$ & 5 & 1 & 0.16 & 48.05 & 0.28 & 45.97 & 1.91 & 0.32 & 0.16 & 0.04 & 0.00 & 96.89 \\
\hline & $\mathrm{Cpx}$ & 24 & 1 & 48.55 & 1.05 & 1.32 & 32.20 & 12.14 & 4.38 & 0.09 & 0.01 & 0.09 & 99.83 \\
\hline \multirow[t]{8}{*}{ 7-sc4-b10 } & Wht & 30 & 8 & $0.16(0.07)$ & $0.05(0.01)$ & $0.16(0.11)$ & $4.66(0.18)$ & $3.89(0.06)$ & $44.26(0.29)$ & $0.20(0.03)$ & $0.01(0.02)$ & $45.56(0.35)$ & 98.96 \\
\hline & Liq1 & 5 & 5 & $12.78(0.83)$ & $2.17(0.53)$ & $3.10(0.24)$ & $22.86(0.55)$ & $11.14(0.48)$ & $14.50(0.48)$ & $0.62(0.12)$ & $0.14(0.03)$ & $31.33(1.02)$ & 98.65 \\
\hline & Liq2 & 8 & 7 & $71.86(1.47)$ & $0.99(0.09)$ & $12.85(0.27)$ & $4.32(0.25)$ & $0.78(0.06)$ & $1.43(0.12)$ & $0.76(0.06)$ & $2.54(0.08)$ & $1.22(0.13)$ & 96.75 \\
\hline & Plag & 22 & 1 & 57.47 & 0.11 & 26.65 & 1.16 & 0.07 & 9.16 & 5.40 & 0.35 & 0.13 & 100.51 \\
\hline & $\mathrm{Mt}$ & 7 & 1 & 0.15 & 16.79 & 4.04 & 71.85 & 2.70 & 0.10 & 0.02 & 0.01 & 0.01 & 95.67 \\
\hline & Ilm & 3 & 1 & 0.143 & 44.631 & 0.343 & 47.969 & 3.134 & 0.133 & 0.000 & 0.000 & 0.025 & 96.379 \\
\hline & Qtz & 10 & 1 & 94.89 & 0.39 & 2.57 & 0.41 & 0.00 & 0.23 & 0.25 & 0.03 & 0.07 & 98.83 \\
\hline & Lpx & 20 & 1 & 50.63 & 0.52 & 1.71 & 25.28 & 20.13 & 0.88 & 0.02 & 0.01 & 0.10 & 99.27 \\
\hline
\end{tabular}




\begin{tabular}{|c|c|c|c|c|c|c|c|c|c|c|c|c|c|}
\hline \multirow[t]{7}{*}{ 7-sc4-85 } & Wht & 11 & 5 & $0.90(0.47)$ & $0.14(0.02)$ & $0.20(0.10)$ & $2.75(0.10)$ & $3.49(0.04)$ & $46.99(0.65)$ & $0.36(0.04)$ & $0.04(0.02)$ & $44.40(0.27)$ & 99.26 \\
\hline & Liq & 14 & 4 & $75.96(4.33)$ & $1.57(1.17)$ & $10.85(0.70)$ & $0.57(0.12)$ & $0.31(0.18)$ & $2.74(1.75)$ & $1.27(0.99)$ & $0.81(0.81)$ & $1.17(0.98)$ & 95.25 \\
\hline & Plag & 32 & 4 & $56.26(0.47)$ & $0.30(0.17)$ & $25.36(1.55)$ & $1.47(0.35)$ & $0.23(0.07)$ & $9.88(0.55)$ & $5.27(0.18)$ & $0.24(0.18)$ & $0.51(0.49)$ & 99.51 \\
\hline & $\mathrm{Mt}$ & 3 & 1 & 0.18 & 11.63 & 1.80 & 77.19 & 2.46 & 0.28 & 0.00 & 0.04 & 0.01 & 93.58 \\
\hline & Ilm & 6 & 1 & 0.72 & 51.08 & 1.10 & 37.68 & 3.01 & 0.42 & 0.01 & 0.04 & 0.00 & 94.05 \\
\hline & Qtz & 5 & 1 & 91.84 & 0.57 & 3.75 & 0.37 & 0.10 & 0.69 & 0.44 & 0.02 & 0.30 & 98.07 \\
\hline & $\mathrm{Cpx}$ & 29 & 1 & 51.30 & 1.05 & 1.80 & 19.57 & 20.26 & 4.40 & 0.13 & 0.07 & 0.47 & 99.06 \\
\hline \multirow{9}{*}{7 -sc4-810 } & Wht & 6 & 7 & $0.69(0.37)$ & $0.11(0.05)$ & $0.22(0.07)$ & $3.15(0.08)$ & $4.59(0.09)$ & $46.00(0.91)$ & $0.27(0.04)$ & $0.04(0.03)$ & $44.00(0.33)$ & 99.09 \\
\hline & Liq1 & 30 & 2 & $17.65(3.31)$ & $1.16(0.15)$ & $4.50(0.17)$ & $12.10(0.76)$ & $14.28(0.55)$ & $17.18(0.63)$ & $1.34(0.31)$ & $0.31(0.12)$ & $33.91(0.63)$ & 102.42 \\
\hline & Liq2 & 10 & 4 & $71.83(0.99)$ & $0.78(0.06)$ & $12.14(0.63)$ & $2.60(0.20)$ & $1.35(0.41)$ & $2.34(0.89)$ & $1.05(0.22)$ & $1.82(0.14)$ & $2.17(0.54)$ & 96.09 \\
\hline & Plag & 30 & 1 & 60.55 & 0.20 & 23.37 & 1.36 & 0.27 & 8.00 & 5.16 & 0.28 & 0.64 & 99.81 \\
\hline & $\mathrm{Mt}$ & 3 & 1 & 0.28 & 6.22 & 3.80 & 76.45 & 3.73 & 0.38 & 0.03 & 0.04 & 0.00 & 90.93 \\
\hline & $\mathrm{Ilm}$ & 5 & 1 & 3.18 & 44.87 & 3.18 & 38.69 & 3.84 & 1.47 & 0.15 & 0.00 & 1.40 & 96.77 \\
\hline & Qtz & 7 & 1 & 86.52 & 0.34 & 7.20 & 0.33 & 0.06 & 1.48 & 1.20 & 0.08 & 0.16 & 97.36 \\
\hline & Lpx & 6 & 4 & $48.11(1.36)$ & 2.07 (1.49) & $3.49(0.51)$ & $16.63(0.84)$ & 24.75 (1.98) & 2.37 (1.09) & $0.12(0.14)$ & $0.10(0.15)$ & $2.55(0.70)$ & 100.17 \\
\hline & Stan & 3 & 4 & $2.27(1.28)$ & $0.13(0.02)$ & $0.57(0.53)$ & $7.23(0.28)$ & $17.59(0.51)$ & $26.35(0.55)$ & $0.14(0.08)$ & $0.06(0.02)$ & $45.04(1.04)$ & 99.38 \\
\hline \multirow[t]{2}{*}{ 2-sc4-b5 } & Wht & 5 & 5 & $0.48(0.67)$ & $0.07(0.04)$ & $0.11(0.17)$ & $3.03(0.19)$ & $3.57(0.15)$ & $47.26(0.33)$ & $0.39(0.09)$ & $0.04(0.05)$ & $44.32(0.65)$ & 99.28 \\
\hline & Liq & 95 & 8 & $47.66(0.38)$ & $4.62(0.10)$ & $11.18(0.15)$ & $14.65(0.15)$ & $4.69(0.12)$ & $10.17(0.19)$ & $2.12(0.18)$ & $0.52(0.02)$ & $4.63(0.10)$ & 100.24 \\
\hline \multirow[t]{5}{*}{ 2-sc4-b10 } & Wht & 20 & 12 & $0.16(0.08)$ & $0.08(0.03)$ & $0.08(0.04)$ & $3.21(0.09)$ & $4.40(0.07)$ & $45.60(0.45)$ & $0.18(0.04)$ & $0.02(0.02)$ & $47.32(1.10)$ & 101.05 \\
\hline & Liq1 & 35 & 8 & $60.28(2.12)$ & $1.86(0.10)$ & $13.46(0.15)$ & $8.53(0.61)$ & $3.51(0.35)$ & $5.01(0.42)$ & $2.33(0.41)$ & $0.63(0.06)$ & $3.88(0.64)$ & 99.49 \\
\hline & Liq2 & 30 & 8 & $40.13(1.40)$ & $3.10(0.09)$ & $10.71(0.43)$ & $15.79(0.41)$ & $8.20(0.49)$ & $9.33(0.26)$ & $1.45(0.10)$ & $0.20(0.02)$ & $11.33(0.86)$ & 100.24 \\
\hline & $\mathrm{Mt}$ & 10 & 5 & $0.12(0.03)$ & $9.84(0.22)$ & $5.41(0.34)$ & $75.22(1.11)$ & $4.11(0.16)$ & $0.17(0.04)$ & $0.02(0.03)$ & $0.011(0.01)$ & $0.03(0.04)$ & 94.93 \\
\hline & Qtz & 5 & 7 & $96.67(0.56)$ & $0.44(0.04)$ & $2.62(0.23)$ & $0.40(0.08)$ & $0.01(0.01)$ & $0.28(0.02)$ & $0.40(0.07)$ & $0.03(0.04)$ & $0.02(0.03)$ & 100.87 \\
\hline \multirow[t]{6}{*}{ 2-sc4-85 } & Wht & 10 & 9 & $0.28(0.06)$ & $0.09(0.05)$ & $0.08(0.04)$ & $2.87(0.15)$ & $3.39(0.06)$ & $46.57(0.26)$ & $0.23(0.03)$ & $0.03(0.01)$ & $47.36(0.56)$ & 100.90 \\
\hline & Liq & 25 & 10 & $66.52(1.00)$ & $2.92(0.14)$ & $11.91(0.28)$ & $7.11(0.90)$ & $1.80(0.13)$ & $5.11(0.34)$ & $2.14(0.14)$ & $1.01(0.05)$ & $1.19(0.15)$ & 99.71 \\
\hline & Plag & 30 & 5 & $58.15(0.46)$ & $0.23(0.04)$ & $25.95(0.31)$ & $0.64(0.09)$ & $0.19(0.03)$ & $9.60(0.30)$ & $5.40(0.13)$ & $0.12(0.03)$ & $0.12(0.06)$ & 100.39 \\
\hline & $\mathrm{Ilm}$ & 4 & 2 & $0.06(0.01)$ & $53.03(0.01)$ & $0.23(0.02)$ & $39.71(0.02)$ & $4.79(0.04)$ & $0.21(0.00)$ & $0.01(0.02)$ & $0.01(0.00)$ & $0(0)$ & 98.05 \\
\hline & Psd & 4 & 5 & $0.13(0.14)$ & $67.37(0.41)$ & $1.42(0.12)$ & $24.48(0.73)$ & $5.14(0.13)$ & $0.23(0.02)$ & $0.01(0.02)$ & $0.02(0.01)$ & $0.03(0.04)$ & 98.83 \\
\hline & $\mathrm{Cpx}$ & 27 & 4 & $51.80(0.89)$ & $1.92(0.14)$ & $1.96(0.33)$ & $13.34(0.91)$ & 20.15 (1.19) & $10.79(1.45)$ & $0.15(0.02)$ & $0.01(0.01)$ & $0.39(0.44)$ & 100.51 \\
\hline \multirow[t]{6}{*}{ 2-sc4-810 } & Wht & 10 & 10 & $1.07(1.46)$ & $0.13(0.11)$ & $0.25(0.30)$ & $2.56(0.66)$ & $5.22(0.68)$ & $44.56(1.63)$ & $0.24(0.06)$ & $0.02(0.03)$ & 46.92 (1.99) & 100.97 \\
\hline & Liq1 & 10 & 5 & $22.80(1.77)$ & $2.02(0.22)$ & $4.43(0.37)$ & $12.87(0.34)$ & $16.62(0.78)$ & $14.59(1.42)$ & $0.82(0.10)$ & $0.08(0.03)$ & $26.79(1.31)$ & 101.03 \\
\hline & Liq2 & 32 & 7 & $69.98(1.68)$ & $1.07(0.13)$ & $11.89(0.52)$ & $4.27(0.71)$ & $2.77(1.14)$ & $2.90(0.58)$ & $0.84(0.25)$ & $1.18(0.07)$ & $2.27(0.94)$ & 97.17 \\
\hline & Plag & 40 & 3 & $60.22(0.22)$ & $0.38(0.07)$ & $22.90(0.79)$ & $1.87(0.35)$ & $0.88(0.42)$ & $8.19(0.28)$ & $4.82(0.35)$ & $0.38(0.09)$ & $1.10(0.60)$ & 100.74 \\
\hline & $\mathrm{Mt}$ & 3 & 2 & $0.16(0.01)$ & $4.40(0.14)$ & $4.09(0.27)$ & $76.14(1.12)$ & $5.93(0.16)$ & $0.23(0.03)$ & $0.00(0.01)$ & $0.01(0.01)$ & $0.05(0.01)$ & 91.01 \\
\hline & Ilm & 5 & 1 & 0.63 & 46.21 & 2.46 & 42.23 & 4.11 & 0.87 & 0.01 & 0.05 & 0.53 & 97.10 \\
\hline \multirow[t]{3}{*}{3 -sc4-b5 } & Wht & 5 & 4 & $0.31(0.24)$ & $0.07(0.03)$ & $0.10(0.12)$ & $3.02(0.11)$ & $3.68(0.01)$ & $46.99(0.33)$ & $0.27(0.02)$ & $0.01(0.01)$ & $45.43(0.94)$ & 99.89 \\
\hline & Liq & 80 & 3 & $46.65(0.25)$ & $4.81(0.11)$ & $10.85(0.06)$ & $15.42(0.09)$ & $4.95(0.60)$ & $10.05(0.14)$ & $2.12(0.06)$ & $0.51(0.02)$ & $4.36(0.07)$ & 99.71 \\
\hline & Plag & 15 & 4 & $57.19(0.23)$ & $0.16(0.01)$ & $27.28(0.16)$ & $0.70(0.07)$ & $0.51(0.50)$ & $9.91(0.20)$ & $5.42(0.13)$ & $0.18(0.01)$ & $0.06(0.02)$ & 101.40 \\
\hline \multirow[t]{6}{*}{ 3-sc4-b10 } & Wht & 15 & 10 & $0.11(0.04)$ & $0.07(0.02)$ & $0.08(0.03)$ & $2.45(0.20)$ & $5.09(0.08)$ & $45.49(0.21)$ & $0.18(0.02)$ & $0.02(0.02)$ & $46.43(0.36)$ & 99.92 \\
\hline & Liq1 & 15 & 8 & $17.52(0.45)$ & $2.03(0.09)$ & $4.11(0.14)$ & $12.04(0.55)$ & $17.81(0.46)$ & $15.10(0.58)$ & $0.81(0.13)$ & $0.09(0.03)$ & $29.78(0.69)$ & 99.28 \\
\hline & Liq2 & 20 & 7 & $66.62(1.26)$ & $1.08(0.05)$ & $12.57(0.18)$ & $4.35(0.45)$ & $2.25(0.34)$ & $2.79(0.52)$ & $0.84(0.11)$ & $1.43(0.14)$ & $1.85(0.59)$ & 93.78 \\
\hline & Plag & 35 & 6 & $52.86(0.35)$ & $0.13(0.01)$ & $26.87(0.60)$ & $1.46(0.09)$ & $0.27(0.13)$ & $10.46(0.25)$ & $4.84(0.14)$ & $0.19(0.01)$ & $0.42(0.24)$ & 97.50 \\
\hline & $\mathrm{Mt}$ & 10 & 5 & $0.05(0.02)$ & $21.65(0.37)$ & $1.27(0.04)$ & $66.76(0.59)$ & $3.28(0.22)$ & $0.29(0.06)$ & $0.01(0.01)$ & $0.01(0.01)$ & $0.01(0.02)$ & 93.33 \\
\hline & Qtz & 5 & 4 & $92.15(0.24)$ & $0.49(0.03)$ & $2.5(0.32)$ & $0.41(0.05)$ & $0.01(0.02)$ & $0.20(0.03)$ & $0.26(0.10)$ & $0.03(0.01)$ & $0.04(0.01)$ & 96.10 \\
\hline \multirow[t]{2}{*}{$3-s c 4-85$} & Wht & 5 & 7 & $1.06(0.81)$ & $0.12(0.04)$ & $0.21(0.18)$ & $2.60(0.09)$ & $3.67(0.05)$ & $46.14(0.38)$ & $0.29(0.05)$ & $0.02(0.01)$ & $44.53(0.63)$ & 98.64 \\
\hline & Liq & 36 & 8 & $60.00(1.51)$ & $2.69(0.15)$ & $11.73(0.08)$ & $8.13(0.46)$ & $2.28(0.17)$ & $5.71(0.44)$ & $2.06(0.36)$ & $0.88(0.07)$ & $1.44(0.26)$ & 94.93 \\
\hline
\end{tabular}


Table 3 (continued)

\begin{tabular}{|c|c|c|c|c|c|c|c|c|c|c|c|c|c|}
\hline Run no. & Phase $^{\mathrm{a}}$ & $\%$ modal & $\begin{array}{l}\text { Number } \\
\text { of analyses }\end{array}$ & $\mathrm{SiO}_{2}$ & $\mathrm{TiO}_{2}$ & $\mathrm{Al}_{2} \mathrm{O}_{3}$ & $\mathrm{FeO}$ & $\mathrm{MgO}$ & $\mathrm{CaO}$ & $\mathrm{Na}_{2} \mathrm{O}$ & $\mathrm{K}_{2} \mathrm{O}$ & $\mathrm{P}_{2} \mathrm{O}_{5}$ & Total \\
\hline$\overline{\text { Plag }}$ & 28 & 4 & $52.58(0.63)$ & $0.19(0.02)$ & $26.32(0.43)$ & $1.27(0.17)$ & $0.21(0.02)$ & $10.27(0.42)$ & $5.05(0.19)$ & $0.12(0.00)$ & $0.13(0.14)$ & 96.15 & \\
\hline Psd & 3 & 3 & $3.25(3.09)$ & $57.89(1.47)$ & $1.85(0.30)$ & $29.49(0.88)$ & $5.02(0.10)$ & $0.7(0.18)$ & $0.05(0.04)$ & $0.06(0.06)$ & $0.08(0.04)$ & 98.39 & \\
\hline Cpx & 28 & 3 & $50.18(1.01)$ & $1.06(0.25)$ & $1.63(0.32)$ & $13.01(1.03)$ & $23.68(1.57)$ & $6.95(1.94)$ & $0.13(0.08)$ & $0.03(0.02)$ & $0.13(0.12)$ & 96.81 & \\
\hline \multirow[t]{7}{*}{3 -sc4-810 } & Wht & 6 & 8 & $0.19(0.14)$ & $0.09(0.04)$ & $0.13(0.08)$ & $1.83(0.09)$ & $5.32(0.07)$ & $45.31(0.27)$ & $0.20(0.02)$ & $0.02(0.01)$ & $45.11(0.24)$ & 98.18 \\
\hline & Liq1 & 6 & 8 & $21.29(1.47)$ & $1.74(0.24)$ & $4.51(0.39)$ & $10.96(0.63)$ & 19.09 (1.44) & $13.42(1.24)$ & $0.81(0.06)$ & $0.08(0.02)$ & $26.26(1.19)$ & 98.16 \\
\hline & Liq2 & 28 & 8 & $67.41(1.16)$ & $1.02(0.05)$ & $12.51(0.34)$ & $3.90(0.15)$ & $2.51(0.33)$ & $2.85(0.40)$ & $2.10(0.67)$ & $1.23(0.07)$ & $1.78(0.36)$ & 95.32 \\
\hline & Plag & 40 & 3 & $53.53(2.09)$ & $0.17(0.07)$ & $25.93(0.77)$ & $1.81(0.22)$ & $0.52(0.46)$ & $9.87(0.27)$ & $5.04(0.37)$ & $0.12(0.02)$ & $0.70(0.70)$ & 97.68 \\
\hline & $\mathrm{Mt}$ & 5 & 2 & $0.10(0.01)$ & $3.15(0.09)$ & $4.39(0.02)$ & $77.46(0.14)$ & $7.62(0.07)$ & $0.21(0.04)$ & $0.03(0.01)$ & $0.01(0.02)$ & $0.01(0.02)$ & 92.98 \\
\hline & $\mathrm{Ilm}$ & 5 & 2 & $0.11(0.03)$ & $42.34(0.14)$ & $2.00(0.00)$ & $45.10(0.69)$ & $2.95(0.02)$ & $0.31(0.03)$ & $0.00(0.00)$ & $0.01(0.01)$ & $0.01(0.01)$ & 92.83 \\
\hline & Qtz & 10 & 3 & $89.67(1.68)$ & $0.41(0.03)$ & $3.80(0.27)$ & $0.35(0.09)$ & $0.01(0.02)$ & $0.42(0.05)$ & $0.26(0.05)$ & $0.02(0.01)$ & $0.04(0.02)$ & 94.98 \\
\hline \multicolumn{14}{|l|}{$F M Q+0.5$} \\
\hline \multirow[t]{5}{*}{ 4-sc4-b5 } & Wht & 5 & 7 & $0.98(1.52)$ & $0.07(0.04)$ & $0.54(0.74)$ & $3.19(0.22)$ & $3.46(0.17)$ & $45.68(1.51)$ & $0.21(0.11)$ & $0.02(0.03)$ & $43.46(0.77)$ & 97.60 \\
\hline & Liq & 65 & 10 & $48.98(1.53)$ & $4.38(0.18)$ & $11.08(0.45)$ & $15.43(0.70)$ & $4.32(0.19)$ & $9.23(0.32)$ & $2.09(0.13)$ & $0.64(0.04)$ & $3.80(0.36)$ & 99.94 \\
\hline & Plag & 17 & 1 & 58.03 & 0.14 & 26.63 & 0.81 & 0.13 & 9.36 & 5.88 & 0.24 & 0.03 & 101.25 \\
\hline & $\mathrm{Ilm}$ & 3 & 3 & $0.03(0.01)$ & $48.48(0.92)$ & $0.32(0.01)$ & $44.55(0.94)$ & $3.91(0.33)$ & $0.27(0.03)$ & $0.01(0.02)$ & $0.03(0.03)$ & $0.01(0.02)$ & 97.61 \\
\hline & Cpx & 10 & 1 & 52.00 & 0.80 & 1.00 & 23.39 & 19.16 & 4.96 & 0.08 & 0.02 & 0.06 & 101.46 \\
\hline \multirow[t]{6}{*}{ 4-sc4-b10 } & Wht & 5 & 6 & $0.09(0.03)$ & $0.04(0.04)$ & $0.05(0.02)$ & $3.78(0.13)$ & $4.02(0.12)$ & $45.54(0.37)$ & $0.20(0.01)$ & $0.00(0.01)$ & $44.45(0.51)$ & 98.17 \\
\hline & Liq1 & 29 & 8 & $34.38(0.46)$ & $3.79(0.04)$ & $7.80(0.15)$ & $20.58(0.25)$ & $8.39(0.21)$ & $10.31(0.14)$ & $1.27(0.04)$ & $0.21(0.03)$ & $13.16(0.36)$ & 99.89 \\
\hline & Liq2 & 29 & 8 & $61.22(1.32)$ & $1.96(0.06)$ & $13.36(0.17)$ & $9.33(0.50)$ & $2.61(0.38)$ & $4.45(0.22)$ & $1.52(0.07)$ & $1.26(0.06)$ & $3.09(0.31)$ & 98.80 \\
\hline & Plag & 10 & 1 & $58.12(0.13)$ & $0.14(0.01)$ & $27.04(0.07)$ & $1.00(0.06)$ & $0.03(0.01)$ & $9.71(0.07)$ & $5.15(0.70)$ & $0.19(0.02)$ & $0.12(0.02)$ & 101.49 \\
\hline & $\mathrm{Mt}$ & 5 & 3 & $0.03(0.02)$ & $16.44(0.35)$ & $3.34(0.03)$ & $71.49(0.68)$ & $3.64(0.13)$ & $0.10(0.05)$ & $0.02(0.03)$ & $0.00(0.01)$ & $0(0)$ & 95.07 \\
\hline & $\mathrm{Ilm}$ & 2 & 1 & 0.83 & 48.72 & 1.86 & 39.45 & 3.72 & 0.40 & 0.02 & 0.00 & 0.04 & 95.03 \\
\hline \multirow[t]{6}{*}{$4-s c 4-85$} & Wht & 10 & 7 & $0.78(0.83)$ & $0.12(0.05)$ & $0.10(0.13)$ & $3.09(0.16)$ & $3.33(0.07)$ & $46.12(0.50)$ & $0.28(0.04)$ & $0.03(0.03)$ & $44.74(0.68)$ & 98.58 \\
\hline & Liq & 30 & 8 & $68.27(1.27)$ & $1.70(0.06)$ & $11.62(0.19)$ & $5.47(0.56)$ & $0.99(0.10)$ & $3.14(0.28)$ & $1.73(0.14)$ & $1.50(0.06)$ & $0.52(0.08)$ & 94.95 \\
\hline & Plag & 30 & 4 & $54.93(0.85)$ & $0.24(0.06)$ & $25.63(0.63)$ & $1.30(0.26)$ & $0.22(0.05)$ & $9.57(0.47)$ & $4.54(0.13)$ & $0.16(0.07)$ & $0.09(0.03)$ & 96.69 \\
\hline & Ilm & 5 & 3 & $1.62(0.43)$ & $54.99(1.19)$ & $1.83(0.65)$ & $31.41(2.07)$ & $4.03(0.90)$ & $0.55(0.05)$ & $0.10(0.11)$ & $0.02(0.02)$ & $0.03(0.02)$ & 94.58 \\
\hline & Qtz & 5 & 2 & $90.02(1.97)$ & $0.61(0.08)$ & $2.95(0.05)$ & $0.57(0.35)$ & $0.06(0.06)$ & $0.52(0.15)$ & $0.42(0.11)$ & $0.06(0.07)$ & $0.08(0.08)$ & 95.29 \\
\hline & Cpx & 20 & 3 & $47.49(0.15)$ & $1.81(0.07)$ & $2.48(0.14)$ & $10.91(0.43)$ & $19.15(0.97)$ & $14.33(1.13)$ & $0.27(0.06)$ & $0(0)$ & $0.91(0.21)$ & 97.34 \\
\hline \multirow[t]{8}{*}{ 4-sc4-810 } & Wht & 10 & 7 & $0.22(0.06)$ & $0.09(0.01)$ & $0.02(0.04)$ & $2.96(0.13)$ & $4.79(0.13)$ & $45.18(0.41)$ & $0.22(0.03)$ & $0.02(0.03)$ & $46.21(0.29)$ & 99.70 \\
\hline & Liq1 & 9 & 8 & $67.95(1.92)$ & $1.06(0.17)$ & 12.05 & $3.66(0.17)$ & $1.37(0.76)$ & $2.57(1.66)$ & $1.00(0.46)$ & $1.36(0.12)$ & $1.93(1.41)$ & 92.95 \\
\hline & Liq2 & 20 & 3 & $14.17(1.39)$ & $1.75(0.28)$ & $3.36(0.32)$ & $16.65(0.71)$ & $15.74(1.85)$ & $14.54(1.52)$ & $0.71(0.08)$ & $0.08(0.01)$ & $32.25(1.52)$ & 99.23 \\
\hline & Plag & 45 & 2 & $55.96(0.20)$ & $0.21(0.04)$ & $25.07(0.11)$ & $1.40(0.05)$ & $0.17(0.01)$ & $8.77(0.03)$ & $5.18(0.01)$ & $0.26(0.07)$ & $0.43(0.14)$ & 97.45 \\
\hline & $\mathrm{Mt}$ & 3 & 4 & $1.05(1.69)$ & $6.72(0.20)$ & $4.21(0.22)$ & $76.77(0.70)$ & $4.13(0.37)$ & $0.35(0.15)$ & $0.06(0.10)$ & $0.02(0.02)$ & $0.07(0.06)$ & 93.36 \\
\hline & Ilm & 5 & 1 & 0.83 & 48.72 & 1.86 & 39.45 & 3.72 & 0.40 & 0.02 & 0.00 & 0.04 & 95.03 \\
\hline & Lpx & 5 & 8 & $50.03(0.83)$ & $0.69(0.13)$ & $3.01(0.35)$ & $15.29(1.15)$ & $27.44(1.15)$ & $0.91(0.29)$ & $0.04(0.02)$ & $0.02(0.02)$ & $0.81(0.54)$ & 98.23 \\
\hline & Stan & 3 & 3 & $0.35(0.12)$ & $0.10(0.02)$ & $0.01(0.01)$ & $6.93(0.07)$ & $17.95(0.15)$ & $26.78(0.13)$ & $0.08(0.02)$ & $0(0)$ & $48.24(0.54)$ & 100.44 \\
\hline \multicolumn{14}{|l|}{$F M Q+1.5$} \\
\hline \multirow[t]{5}{*}{ 5-sc4-b5 } & Wht & 5 & 7 & $0.23(0.05)$ & $0.07(0.03)$ & $0.01(0.03)$ & $3.02(0.15)$ & $3.46(0.04)$ & $46.43(0.36)$ & $0.17(0.03)$ & $0.01(0.01)$ & $44.94(0.33)$ & 98.36 \\
\hline & Liq & 10 & 5 & $65.09(1.35)$ & $1.24(0.10)$ & 11.75 & $4.93(0.23)$ & $0.91(0.09)$ & $3.06(0.56)$ & $0.71(0.24)$ & $1.89(0.17)$ & $0.51(0.08)$ & 90.09 \\
\hline & Plag & 43 & 5 & $53.81(2.18)$ & $0.16(0.04)$ & $25.25(0.69)$ & $1.83(0.11)$ & $0.21(0.04)$ & $9.84(0.34)$ & $2.94(1.26)$ & $0.20(0.03)$ & $0.20(0.20)$ & 94.44 \\
\hline & $\mathrm{Mt}$ & 10 & 3 & $0.11(0.09)$ & $11.00(0.44)$ & $2.01(0.14)$ & $76.02(0.40)$ & $2.66(0.04)$ & $0.26(0.11)$ & $0(0)$ & $0.02(0.01)$ & $0.01(0.02)$ & 92.09 \\
\hline & $\mathrm{Ilm}$ & 2 & 3 & $0.10(0.01)$ & $32.63(0.31)$ & $0.48(0.08)$ & $56.56(0.97)$ & $2.29(0.08)$ & $0.41(0.08)$ & $0.01(0.01)$ & $0.02(0.02)$ & $0.06(0.09)$ & 92.55 \\
\hline
\end{tabular}




\begin{tabular}{|c|c|c|c|c|c|c|c|c|c|c|c|c|c|}
\hline & Qtz & 5 & 3 & $84.82(0.53)$ & $0.57(0.31)$ & $2.51(0.41)$ & $0.54(0.17)$ & $0.02(0.02)$ & $0.31(0.09)$ & $0.10(0.03)$ & $0.01(0.01)$ & $0.05(0.08)$ & 88.93 \\
\hline & Cpx & 25 & 3 & $45.14(0.24)$ & $1.19(0.10)$ & $2.46(0.16)$ & $14.18(0.70)$ & $19.00(0.80)$ & $11.34(0.47)$ & $0.12(0.03)$ & $0.01(0.01)$ & $0.45(0.08)$ & 93.89 \\
\hline \multirow[t]{9}{*}{ 5-sc4-b10 } & Wht & 10 & 9 & $0.21(0.12)$ & $0.05(0.04)$ & $0.15(0.06)$ & $2.92(0.21)$ & $4.91(0.13)$ & $44.72(0.46)$ & $0.23(0.03)$ & $0.02(0.01)$ & $44.02(0.58)$ & 97.23 \\
\hline & Liq1 & 10 & 8 & $71.59(1.15)$ & $0.76(0.04)$ & $12.75(0.33)$ & $4.09(0.37)$ & $1.69(0.26)$ & $2.17(0.31)$ & $0.88(0.12)$ & $1.93(0.10)$ & $1.31(0.57)$ & 97.17 \\
\hline & Liq2 & 15 & 7 & $18.79(0.62)$ & $1.62(0.10)$ & $4.08(0.20)$ & $14.58(0.94)$ & $15.83(0.48)$ & $14.51(1.27)$ & $0.81(0.07)$ & $0.14(0.03)$ & $26.74(0.74)$ & 97.09 \\
\hline & Plag & 25 & 1 & 56.38 & 0.08 & 27.02 & 1.17 & 0.10 & 9.52 & 5.41 & 0.21 & 0.12 & 100.00 \\
\hline & $\mathrm{Mt}$ & 10 & 3 & $0.56(0.76)$ & $9.01(0.09)$ & $3.98(0.23)$ & $72.69(1.03)$ & $5.41(0.05)$ & $0.29(0.06)$ & $0.01(0.02)$ & $0.02(0.03)$ & $0.02(0.02)$ & 92.00 \\
\hline & Ilm & 5 & 2 & $0.05(0.06)$ & $29.43(1.3)$ & $0.65(0.04)$ & $57.41(1.7)$ & $4.07(0.27)$ & $0.38(0.01)$ & $0.02(0.03)$ & $0.01(0.02)$ & $0.08(0.05)$ & 92.10 \\
\hline & Qtz & 5 & 1 & 96.22 & 0.29 & 2.44 & 0.43 & 0.03 & 0.20 & 0.29 & 0.07 & 0.00 & 99.97 \\
\hline & Lpx & 15 & 3 & $51.65(0.64)$ & $0.46(0.05)$ & $3.24(0.26)$ & $14.16(1.76)$ & $28.03(1.12)$ & $1.18(0.64)$ & $0.01(0.01)$ & $0.01(0.02)$ & $1.07(0.87)$ & 99.81 \\
\hline & Stan & 5 & 3 & $0.42(0.23)$ & $0.04(0.03)$ & $0.17(0.09)$ & $6.86(0.22)$ & $17.94(0.22)$ & $26.33(0.40)$ & $0.06(0.02)$ & $0.01(0.01)$ & $45.97(0.04)$ & 97.80 \\
\hline \multirow[t]{6}{*}{5 -sc4-85 } & Wht & 20 & 5 & $1.66(0.72)$ & $0.09(0.02)$ & $0.46(0.33)$ & $2.34(0.08)$ & $3.74(0.05)$ & $46.12(0.25)$ & $0.34(0.04)$ & $0.02(0.02)$ & $43.71(0.79)$ & 98.47 \\
\hline & Liq & 7 & 3 & $74.34(0.68)$ & $1.12(0.67)$ & $11.62(0.76)$ & $2.60(0.31)$ & $0.84(0.15)$ & $2.01(0.39)$ & $0.56(0.26)$ & $1.15(0.07)$ & $0.63(0.47)$ & 94.87 \\
\hline & Plag & 35 & 3 & 56.66 & 0.08 & $25.98(1.16)$ & 1.74 & $0.19(0.06)$ & 9.34 & $5.22(0.47)$ & 0.10 & 0.15 & 99.47 \\
\hline & Mt & 7 & 1 & 2.00 & 0.03 & 1.16 & 0.21 & 0.06 & 0.94 & 0.47 & 0.03 & 0.08 & 0.74 \\
\hline & Psd & 3 & 1 & 7.41 & 38.50 & 2.41 & 38.13 & 2.95 & 0.63 & 0.12 & 0.13 & 0.03 & 90.30 \\
\hline & Cpx & 25 & 1 & 51.06 & 0.43 & 2.17 & 14.58 & 19.54 & 9.52 & 0.25 & 0.01 & 1.34 & 98.89 \\
\hline \multirow[t]{9}{*}{5 -sc4-810 } & Wht & 12 & 7 & $0.51(0.39)$ & $0.10(0.02)$ & $0.15(0.11)$ & $2.78(0.21)$ & $4.69(0.33)$ & $44.96(0.76)$ & $0.25(0.06)$ & $0.02(0.02)$ & $44.39(0.53)$ & 97.83 \\
\hline & Liq1 & 10 & 3 & $6.71(0.43)$ & $1.26(0.59)$ & $1.29(0.12)$ & $12.98(0.60)$ & $17.72(0.58)$ & $17.85(0.83)$ & $0.79(0.14)$ & $0.04(0.01)$ & $38.52(0.90)$ & 97.15 \\
\hline & Liq2 & 8 & 6 & $72.18(1.43)$ & $0.93(0.08)$ & $12.47(0.68)$ & $3.16(0.17)$ & $1.42(0.33)$ & $2.55(0.77)$ & $0.94(0.19)$ & $1.41(0.07)$ & $2.35(0.80)$ & 97.40 \\
\hline & Plag & 40 & 1 & 54.53 & 0.04 & 25.23 & 1.62 & 0.70 & 8.65 & 5.45 & 0.14 & 1.72 & 98.07 \\
\hline & $\mathrm{Mt}$ & 5 & 1 & 6.12 & 2.50 & 6.15 & 69.77 & 4.69 & 1.28 & 0.17 & 0.03 & 0.40 & 91.09 \\
\hline & Ilm & 8 & 1 & 0.83 & 38.15 & 2.46 & 40.81 & 3.42 & 0.55 & 0.00 & 0.01 & 0.16 & 86.40 \\
\hline & Qtz & 10 & 1 & 92.74 & 0.38 & 4.06 & 0.21 & 0.01 & 0.46 & 0.35 & 0.01 & 0.05 & 98.28 \\
\hline & Lpx & 8 & 2 & $53.03(1.62)$ & $0.69(0.05)$ & $2.92(0.03)$ & $13.78(0.23)$ & $28.30(0.10)$ & $0.72(0.05)$ & $0.03(0.02)$ & $0.03(0.03)$ & $0.54(0.33)$ & 100.04 \\
\hline & Stan & 4 & 2 & $0.84(0.68)$ & $0.08(0.04)$ & $0.19(0.17)$ & $6.27(0.09)$ & $18.45(0.07)$ & $26.26(0.34)$ & $0.11(0.02)$ & $0.01(0.01)$ & $45.92(0.55)$ & 98.13 \\
\hline \multicolumn{14}{|l|}{$F M Q+5.5$} \\
\hline \multirow[t]{7}{*}{ 8-sc4-b5 } & Wht & 15 & 5 & $1.01(0.67)$ & $0.12(0.03)$ & $0.27(0.28)$ & $1.43(0.14)$ & $4.36(0.05)$ & $47.90(0.29)$ & $0.31(0.04)$ & $0.04(0.01)$ & $44.26(1.04)$ & 99.70 \\
\hline & Liq & 5 & 3 & $74.75(0.72)$ & $1.20(0.19)$ & $11.61(0.81)$ & $2.99(0.24)$ & $1.30(1.00)$ & $2.54(0.19)$ & $1.28(0.23)$ & $1.64(0.13)$ & $0.61(0.37)$ & 97.91 \\
\hline & Plag & 40 & 1 & 65.72 & 3.49 & 10.22 & 9.79 & 0.96 & 2.39 & 2.44 & 1.94 & 1.03 & 97.99 \\
\hline & $\mathrm{Mt}$ & 13 & 1 & 0.14 & 17.95 & 0.83 & 66.70 & 3.83 & 0.33 & 0.00 & 0.01 & 0.04 & 89.83 \\
\hline & $\mathrm{Ilm}$ & 7 & 1 & 2.99 & 41.02 & 2.03 & 42.63 & 3.51 & 0.66 & 0.02 & 0.08 & 0.27 & 93.20 \\
\hline & Qtz & 5 & 1 & 86.22 & 0.59 & 5.24 & 1.13 & 0.21 & 2.41 & 0.88 & 0.07 & 1.02 & 97.76 \\
\hline & $\mathrm{Cpx}$ & 15 & 1 & 57.95 & 1.15 & 5.74 & 6.08 & 13.46 & 14.38 & 0.38 & 0.43 & 0.83 & 100.39 \\
\hline
\end{tabular}

Numbers in parantheses indicate the ecartype.

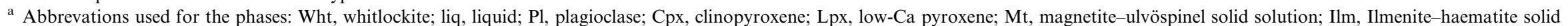
solution; Psd, Pseubroockite- $\mathrm{Fe}_{2} \mathrm{TiO}_{5}$ solid solution; Qtz, quartz; Stan, stanfieldite. 
rect implications for natural systems, immiscibility has the consequence that liquid compositions are extremely variable as detailed below.

In addition to liquid(s) and whitlockite, a wide range of other silicate, oxide and phosphate minerals occur in these experiments. Because the exact compositions and nature of these other phases are not of primary importance in the context of the present study, the number of analyses of each is commonly restricted (Table 3). Furthermore, it is also possible that certain phases were present in the experimental charges but not described, for example, because they were low in abundance and did not intersect the surface exposed for electron-microprobe analysis. However, several broad generalisations can be made concerning the phase relations of the studied compositions. Crystalline silicates are ubiquitous, notably plagioclase $(\mathrm{Pl})$ and at least one pyroxene, either high$\mathrm{Ca}$ clinopyroxene (Cpx) or low-Ca pyroxene (LoCapx). The latter are more common at low oxygen fugacity and high $\mathrm{P}_{2} \mathrm{O}_{5}$, an observation consistent with the results of Toplis et al. (1994a). In addition, a large number of the experiments contained quartz, generally those with additions of $10 \mathrm{wt} \% \mathrm{P}_{2} \mathrm{O}_{5}$. This observation is consistent with the strong increase in the activity coefficient of $\mathrm{Si}$ caused by the incorporation of $\mathrm{P}$ in silicate melts (e.g., Kushiro, 1975; Gan and Hess, 1992; Toplis et al., 1994a). Three different Fe-Ti oxides are described in our experiments; magnetite-ulvöspinel solid solution (Mt), ilmenite-haematite solid solution (Ilm) and pseudobrookite- $-\mathrm{Fe}_{2} \mathrm{TiO}_{5}$ solid solution (Psd). Finally, in addition to whitlockite, certain experiments, particularly those with the highest $\mathrm{P}$ contents, crystallise the $\mathrm{Ca}-$ $\mathrm{Mg}$ phosphate stanfieldite (Stan: $\left(\mathrm{Ca}_{3} \mathrm{Mg}_{3}\left(\mathrm{PO}_{4}\right)\right)_{4}$; Huminicki and Hawthorne, 2002).

\subsection{Variability of liquid composition}

The range of glass compositions observed in this study is extremely wide, covering $10-75 \mathrm{wt} \% \mathrm{SiO}_{2}, 0.5-$ $20 \mathrm{wt}^{\%} \% \mathrm{FeO}^{*}$, and $0.2-40 \mathrm{wt}^{\%} \% \mathrm{P}_{2} \mathrm{O}_{5}$ (Table 3). Before interpreting these values, it is of interest to consider the internal variability of liquid composition within individual experimental charges (in particular for experiments using SC4-8 where the proportion of liquid was low, as mentioned above). First of all we note that the spread in composition between different experiments is much greater than within a single charge, as illustrated in Fig. 2 for the case of $\mathrm{P}_{2} \mathrm{O}_{5}$ in experiments using compositions SC4-8(5) and SC4-8(10). Even so, in experiments with two liquids the range in $\mathrm{P}_{2} \mathrm{O}_{5}$ concentration in the P-rich glass can reach up to $10 \mathrm{wt} \%$ (Fig. 2b), although in terms of relative variability this remains on the order of $\pm 15 \%$ and in this respect is no worse than the $\mathrm{P}_{2} \mathrm{O}_{5}$-poor glass. We also find that average $\mathrm{P}_{2} \mathrm{O}_{5}$ concentration shows no continuous trend as a function of $f \mathrm{O}_{2}$ (Fig. 2a) and that the difference in composition of coexisting liquids is greater at higher
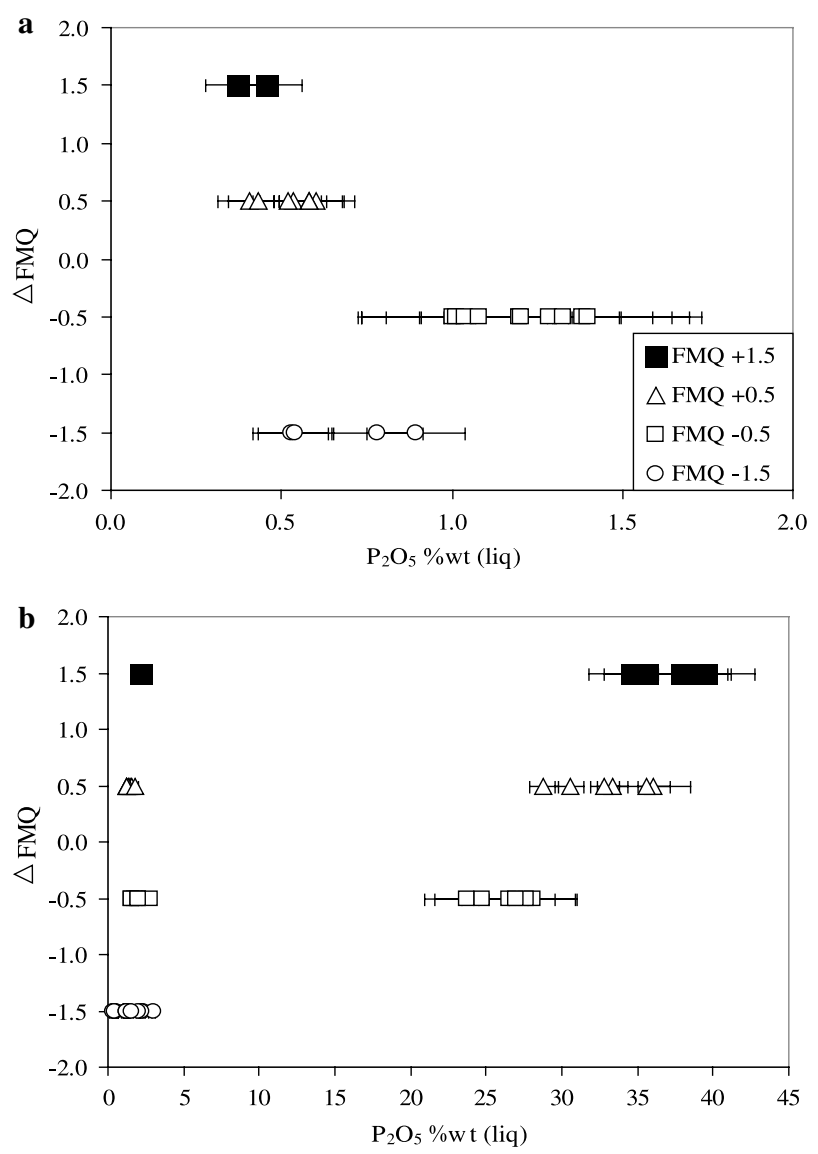

Fig. 2. $\mathrm{P}_{2} \mathrm{O}_{5}$ content of glass (liquid) as a function of $f \mathrm{O}_{2}$ in experiments performed at $1055^{\circ} \mathrm{C}$. (a) Composition SC4-8-5; (b) composition SC4-810 .

oxygen fugacity (Fig. 2b), an observation consistent with the results of Naslund (1983).

Detailed analysis of the spread of liquid composition within a given experimental charge shows that the variability of $\mathrm{P}_{2} \mathrm{O}_{5}$ concentration is systematically correlated with several other compositional parameters. For example, in experiments with a single glass (e.g., bulk composition $\mathrm{SC} 4-8(5))$ the $\mathrm{P}_{2} \mathrm{O}_{5}$ content is inversely correlated with concentration of $\mathrm{SiO}_{2}$ and positively correlated with that of $\mathrm{FeO}^{*}$ and $\mathrm{CaO}$ (Figs. 3a-c). Exactly the same trends are observed in experiments with coexisting liquids (Figs. $3 \mathrm{~d}-\mathrm{f}$ ), both within each individual liquid (most prominent for the P-rich endmember) but also when comparing the P-rich and P-poor glasses. The systematic nature of these correlations, in particular the fact that the variability within one of the endmembers is identical to that observed between coexisting glasses, leads us to conclude that all liquids had reached local equilibrium with whitlockite in our experiments. For this reason, for the data treatment described below we have chosen to consider each individual analysis of liquid composition rather than averages for each experimental charge. 

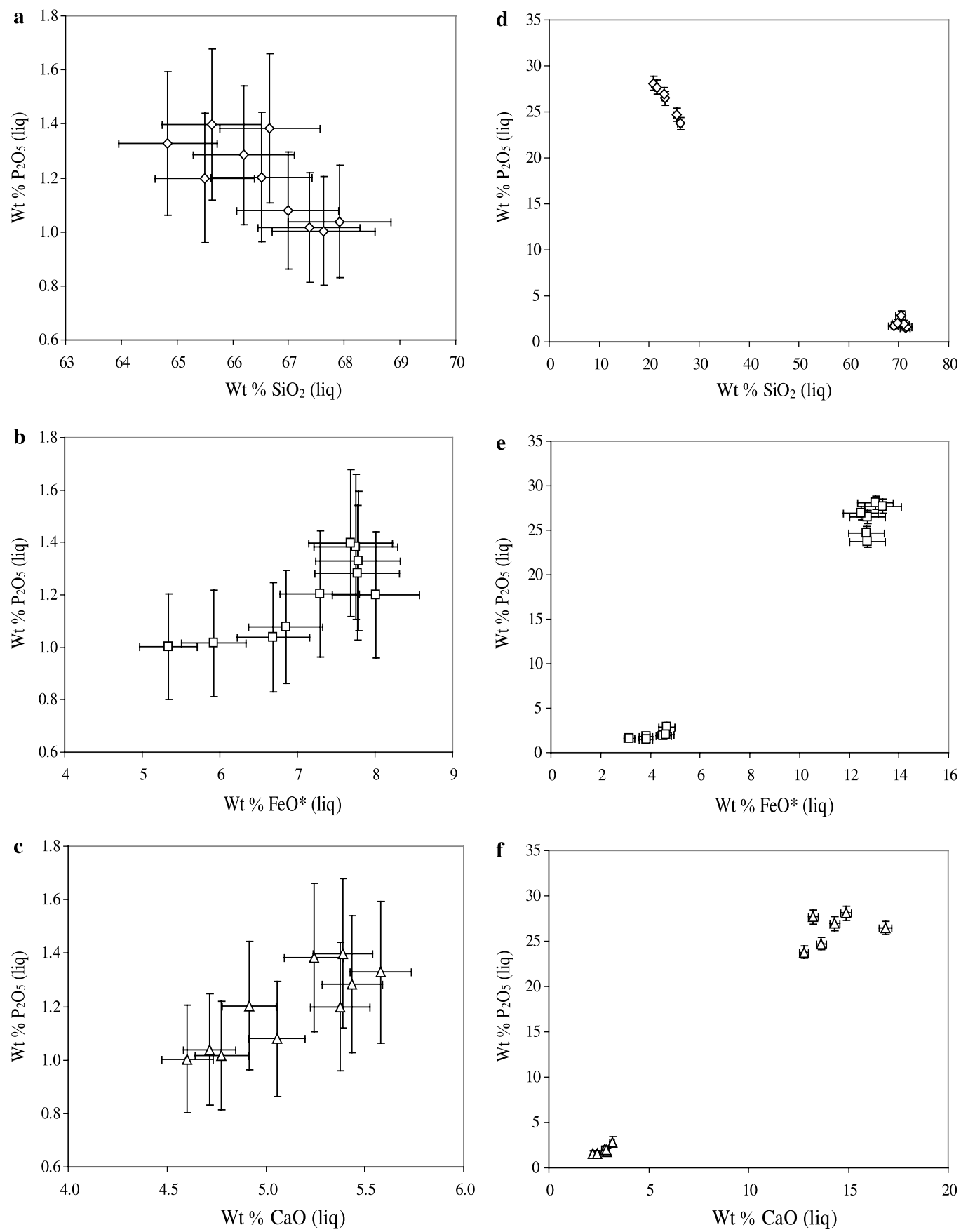

Fig. 3. $\mathrm{SiO}_{2}$, and $\mathrm{FeO}^{*}$ and $\mathrm{CaO}$ concentrations as a function of $\mathrm{P}_{2} \mathrm{O}_{5}$ in liquids of experiments 2-SC4-8-5 (a-c) and 2-SC4-8-10 (d-f) (experiments performed at $1055^{\circ} \mathrm{C}$ and $\triangle \mathrm{FMQ}-0.5$ ).

\section{Discussion}

\subsection{The influence of individual melt components on whitlockite saturation}

\subsubsection{The effect of iron}

To test the hypothesis that Fe content may affect phosphate saturation, the $\mathrm{P}_{2} \mathrm{O}_{5}$ and $\mathrm{FeO}^{*}$ concentrations of our liquids at $1055^{\circ} \mathrm{C}$ have been compared (Fig. 4). Even though there is a reasonable positive correlation between these two parameters at fixed oxygen fugacity (e.g., Figs.
$3 \mathrm{~b}$ and $\mathrm{e})$, when all the data are considered they do not define a single trend (Fig. 4), the scatter being particularly large for the bulk compositions with additions of $10 \mathrm{wt} \%$ $\mathrm{P}_{2} \mathrm{O}_{5}$ (Fig. 4b). However, such dispersion may be expected if it is ferric iron, rather than $\mathrm{FeO}^{*}$, which can stabilise $\mathrm{P}$ in the liquid. The $\mathrm{Fe}_{2} \mathrm{O}_{3}$ concentrations of each liquid have therefore been estimated using the calculation scheme of Kilinc et al. (1983), with an additional term for the effect of $\mathrm{P}_{2} \mathrm{O}_{5}$ taken from Toplis et al. (1994b). However, even when $\mathrm{P}_{2} \mathrm{O}_{5}$ and $\mathrm{Fe}_{2} \mathrm{O}_{3}$ concentrations in the liquids are compared no single trend is apparent (Fig. 5) and there is 
a $1055^{\circ} \mathrm{C}$ isotherm with addition of $5 \mathrm{wt} \% \mathrm{P}_{2} \mathrm{O} 5$

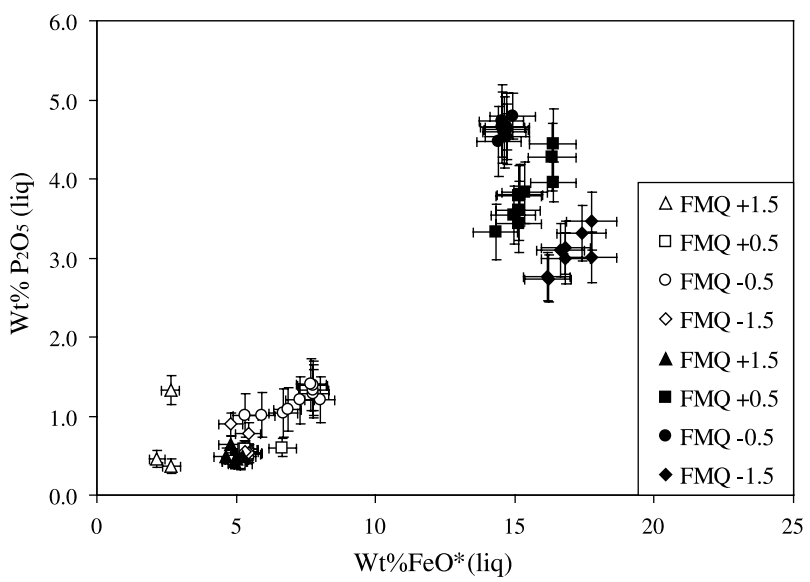

b $1055^{\circ} \mathrm{C}$ isotherm with addition of $10 \mathrm{wt} \% \mathrm{P}_{2} \mathrm{O} 5$

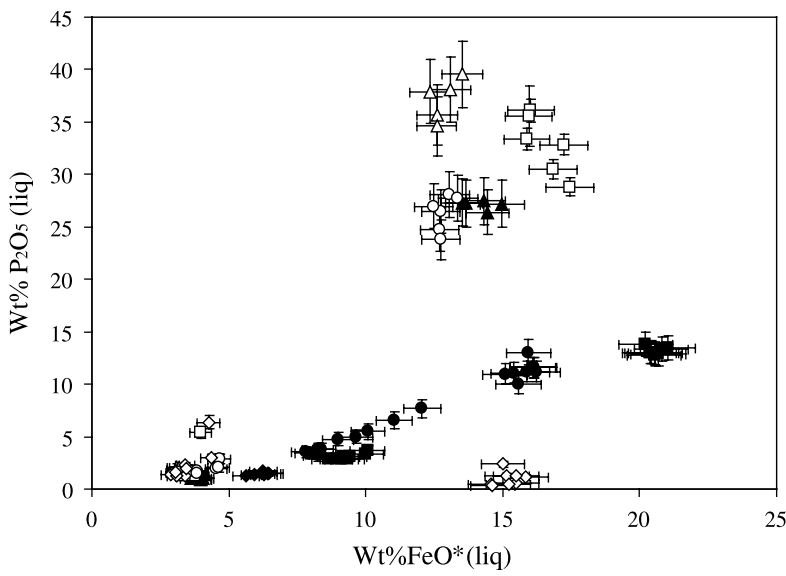

Fig. 4. Covariation of weight percent (wt $\%) \mathrm{P}_{2} \mathrm{O}_{5}$ and $\mathrm{wt}^{\%} \% \mathrm{FeO}^{*}$ of phosphate saturated liquids at $1055^{\circ} \mathrm{C}$. (a) Bulk compositions with $5 \mathrm{wt} \%$ $\mathrm{P}_{2} \mathrm{O}_{5}$. (b) bulk compositions with $10 \mathrm{wt} \% \mathrm{P}_{2} \mathrm{O}_{5}$. Experiments at different oxygen fugacities are distinguished as shown in the key.

a similar level of scatter to that observed for $\mathrm{FeO}^{*}$. It would therefore appear that ferric iron is not the dominant factor controlling phosphate stability and we conclude that some other characteristic(s) of melt composition must be considered to explain the observed variation of the $\mathrm{P}_{2} \mathrm{O}_{5}$ content of whitlockite saturated liquids.

\subsubsection{The effect of silica}

Previous experimental studies have concluded that the $\mathrm{SiO}_{2}$ content of the liquid is one of the dominant factors affecting saturation of crystalline phosphates (Watson, 1979; Harrison and Watson, 1984; Sha, 2000). Our liquids cover a range of $\mathrm{SiO}_{2}$ content even wider than those of previous studies and we too find that there is a good first order anticorrelation of $\mathrm{P}_{2} \mathrm{O}_{5}$ and $\mathrm{SiO}_{2}$, independent of oxygen fugacity and temperature (Fig. 6a). In $\mathrm{SiO}_{2}$-poor liquids, $\mathrm{P}_{2} \mathrm{O}_{5}$ contents are highest, but the overall variation of $\mathrm{P}_{2} \mathrm{O}_{5}$ and $\mathrm{SiO}_{2}$ is non-linear, with $\mathrm{P}_{2} \mathrm{O}_{5}$ concentration flattening off at high $\mathrm{SiO}_{2}$ (Fig. 6a). However, when one considers the data in detail it is apparent that at constant $\mathrm{SiO}_{2}$ content there is considerable variation of $\mathrm{P}_{2} \mathrm{O}_{5}$ concentra- a $1055^{\circ} \mathrm{C}$ isotherm with addition of $5 \mathrm{wt} \% \mathrm{P}_{2} \mathrm{O} 5$

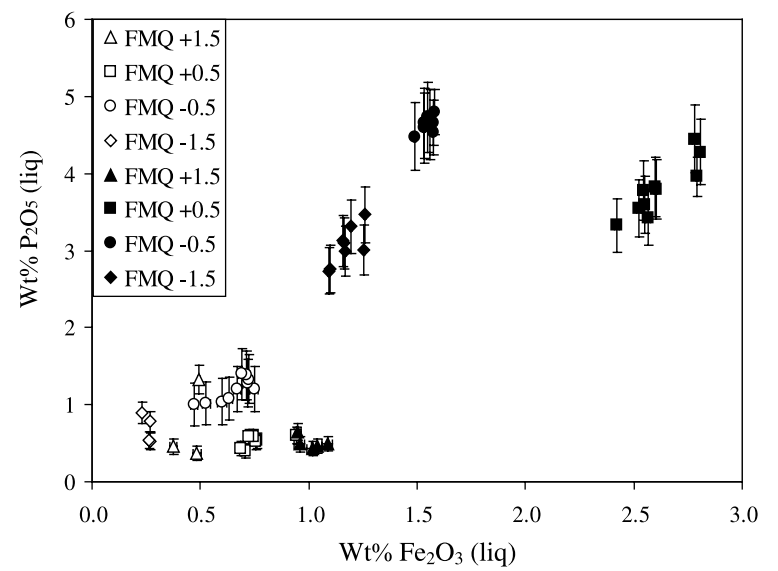

b $1055^{\circ} \mathrm{C}$ isotherm with addition of $10 \mathrm{wt} \% \mathrm{P}_{2} \mathrm{O} 5$

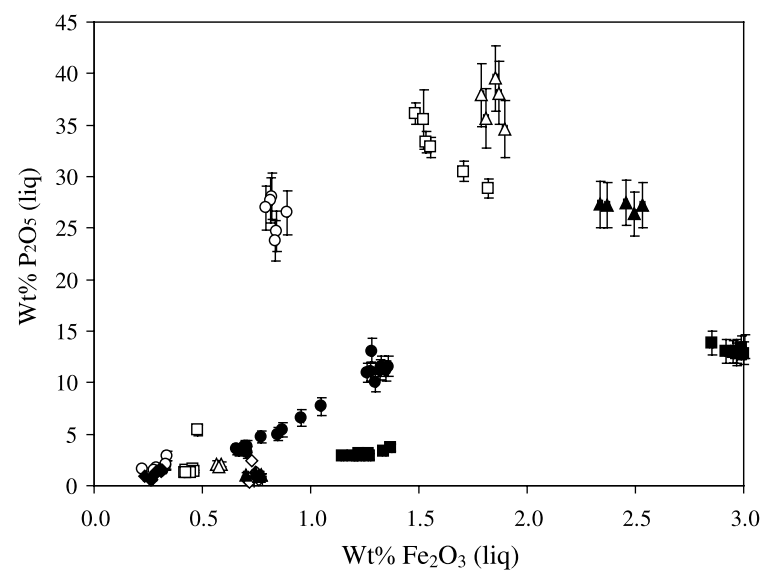

Fig. 5. Covariation of $w t^{0} \% \mathrm{P}_{2} \mathrm{O}_{5}$ and calculated $w t \% \mathrm{Fe}_{2} \mathrm{O}_{3}$ of phosphate saturated liquids at $1055^{\circ} \mathrm{C}$. (a) Bulk compositions with $5 \mathrm{wt} \% \mathrm{P}_{2} \mathrm{O}_{5}$. (b) Bulk compositions with $10 \mathrm{wt} \% \mathrm{P}_{2} \mathrm{O}_{5}$. Experiments at different oxygen fugacities are distinguished as shown in the key.

tion. For example, at $50 \mathrm{wt} \% \mathrm{SiO}_{2}, \mathrm{P}_{2} \mathrm{O}_{5}$ ranges from 3 to $7 \mathrm{wt} \%$, while at $70 \mathrm{wt} \% \mathrm{SiO}_{2}, \mathrm{P}_{2} \mathrm{O}_{5}$ ranges from 0.5 to $3 \mathrm{wt} \%$. Furthermore, the data from experiments without immiscibility appear to define a different trend from data in experiments showing immiscibility. The experimental products without immiscibility are systematically lower in P-content at a given $\mathrm{SiO}_{2}$ content (Fig. 6b). The data of Watson (1979) generally overlap the trend defined by experiments containing only one liquid (Fig. 6b). This is consistent with the fact that no immiscibility was described in those experiments, but is in spite of the facts that liquids of that study were saturated in apatite (rather than whitlockite) and that experiments were performed over a range of temperatures. In conclusion, even though $\mathrm{SiO}_{2}$ content of the liquid would appear to influence phosphate saturation, the dispersion in the data leads us to infer that it is not the only factor.

\subsubsection{The effect of calcium}

Calcium is an essential constituent of both whitlockite and apatite and from a thermodynamic perspective the con- 

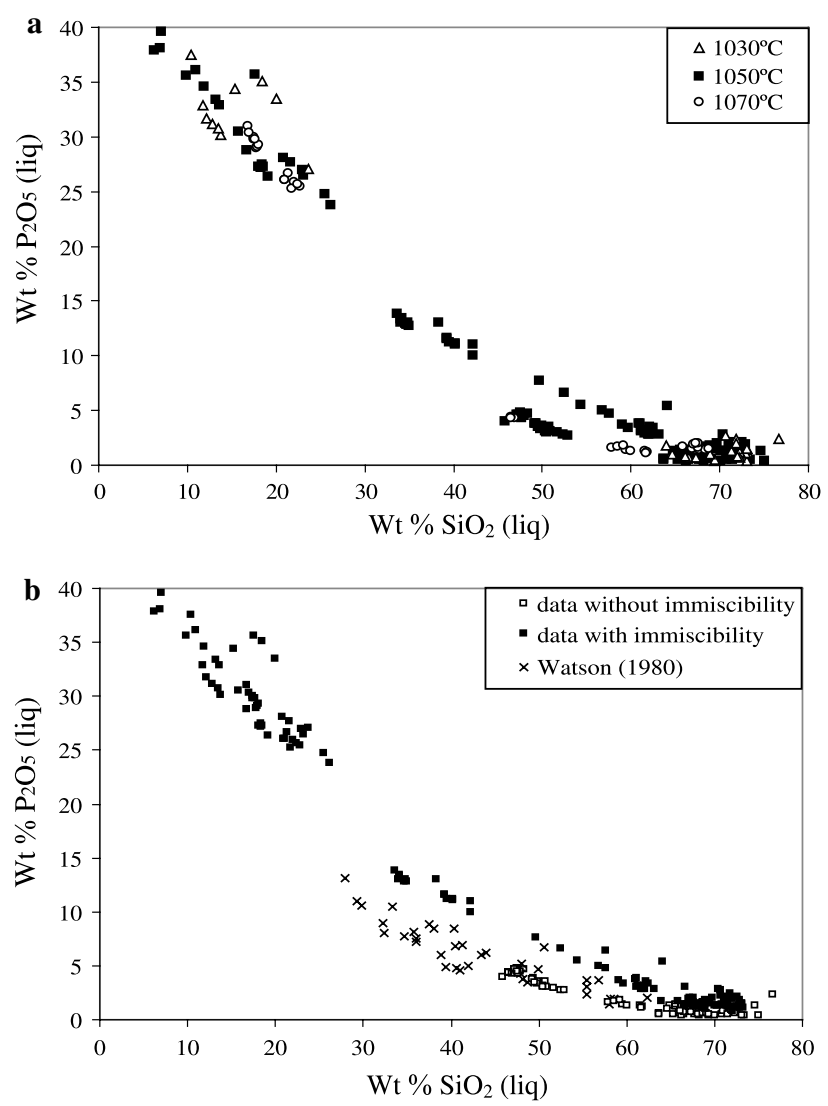

Fig. 6. Covariation of $w t \% \mathrm{P}_{2} \mathrm{O}_{5}$ and $\mathrm{wt} \% \mathrm{SiO}_{2}$ of phosphate saturated liquids. Liquids distinguished by temperature (a) and by the presence or absence of immiscibility (b).

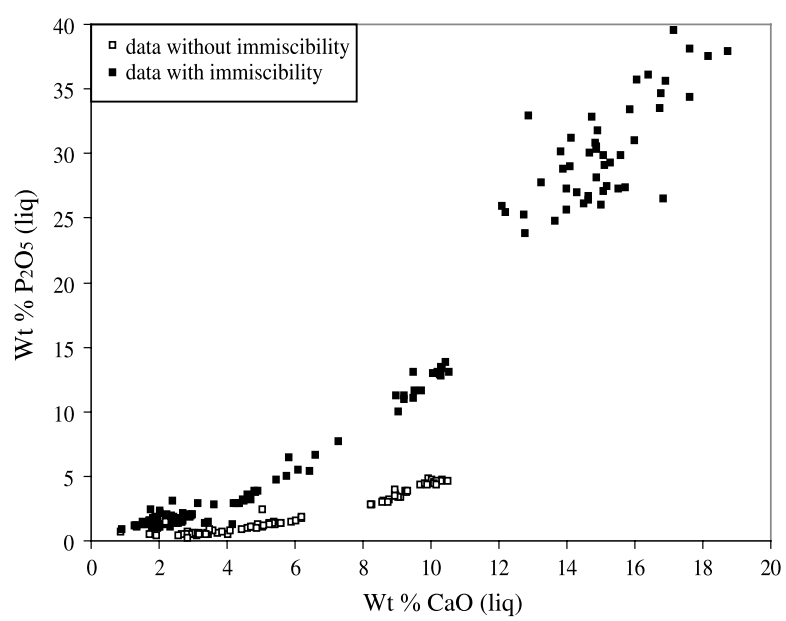

Fig. 7. Covariation of $w t \% \mathrm{CaO}$ and $w t \% \mathrm{P}_{2} \mathrm{O}_{5}$ in liquids at $1055^{\circ} \mathrm{C}$ in experiments with and without immiscibility.

centration of $\mathrm{CaO}$ may be expected to affect the saturation of these minerals. Our data show that concentrations of $\mathrm{P}_{2} \mathrm{O}_{5}$ and $\mathrm{CaO}$ in whitlockite saturated liquids are indeed very well correlated, increasing $\mathrm{CaO}$ content resulting in a highly nonlinear increase of the quantity of $\mathrm{P}_{2} \mathrm{O}_{5}$ necessary to crystallise whitlockite (Fig. 7). For example, for the samples without immiscibility, approximately $1 \mathrm{wt} \% \mathrm{P}_{2} \mathrm{O}_{5}$ is necessary to saturate in whitlockite at $5 \mathrm{wt} \% \mathrm{CaO}$, while $\sim 4.5 \mathrm{wt} \%$ is neces- sary at $10 \mathrm{wt} \% \mathrm{CaO}$ (Fig. 7). However, as for the case of $\mathrm{SiO}_{2}$ previously described, the data from experiments without immiscibility define a distinct trend from data in experiments showing immiscibility, the experiments without immiscibility containing less $\mathrm{P}_{2} \mathrm{O}_{5}$ at saturation at a given $\mathrm{CaO}$ content (Fig. 7).

\subsection{Development of an equation to predict phosphate saturation}

\subsubsection{Whitlockite saturation at fixed temperature}

An alternative approach to understanding the saturation of a crystalline phosphate from silicate liquids is to employ the principles of equilibrium thermodynamics, in particular the notion of an equilibrium constant (or solubility product), as detailed below. In passing we note that although the use of solubility products is widespread when describing crystallisation from aqueous solutions, it is uncommon for equilibria involving silicate melts. However, this approach has been shown to be successful in rationalising solubility data for various minerals such as columbite, hafnon and zircon in granitic liquids (Linnen and Keppler, 1997, 2002).

If we consider the simplified case of saturation of tricalcium phosphate (Mg-, Fe-free whitlockite), one may write the equation:

$3 \mathrm{CaO}^{\text {liq }}+\mathrm{P}_{2} \mathrm{O}_{5}^{\text {liq }}=\mathrm{Ca}_{3}\left(\mathrm{PO}_{4}\right)_{2}{ }^{\mathrm{TCP}}$

The equilibrium constant $(K)$ of this reaction, which should be constant at fixed temperature, may be defined in terms of thermodynamic activities $(a)$ in the following way:

$K_{\mathrm{Ca}_{3}\left(\mathrm{PO}_{4}\right)_{2}}=\left(a_{\mathrm{CaO}}^{\mathrm{liq}}\right)^{3} \times\left(a_{\mathrm{P}_{2} \mathrm{O}_{5}}^{\mathrm{liq}}\right) /\left(a_{\mathrm{Ca}_{3}\left(\mathrm{PO}_{4}\right)_{2}}^{\mathrm{TCP}}\right)$.

For liquids saturated in pure tricalcium phosphate at fixed temperature, the activity of $\mathrm{Ca}_{3}\left(\mathrm{PO}_{4}\right)_{2}$ may be defined as unity, thus, expanding the activities in Eq. (2) in terms of mole fraction $(X)$ and activity coefficient $(\gamma)$ one obtains:

$K_{\mathrm{Ca}_{3}\left(\mathrm{PO}_{4}\right)_{2}}=\left(X_{\mathrm{CaO}}^{\mathrm{liq}}\right)^{3} \times\left(X_{\mathrm{P}_{2} \mathrm{O}_{5}}^{\mathrm{liq}}\right) \times\left(\gamma_{\mathrm{CaO}}^{\mathrm{liq}}\right)^{3} \times\left(\gamma_{\mathrm{P}_{2} \mathrm{O}_{5}}^{\mathrm{liq}}\right)$.

Quantitative application of this equation to our data is potentially compromised by two factors. The first is that the activity coefficients of $\mathrm{CaO}$ and $\mathrm{P}_{2} \mathrm{O}_{5}$ in silicate liquids show complex variations as a function of liquid composition (e.g., Toplis and Schaller, 1998; Libourel, 1999) which will be difficult to model and predict with current thermodynamic models of silicate liquids (Ghiorso et al., 1983). The second is that the phosphates in our experiments are $\mathrm{Mg}$, Fe-bearing whitlockites rather than pure tricalcium phosphate, thus it cannot be assumed that the activity of $\mathrm{Ca}_{3}\left(\mathrm{PO}_{4}\right)_{2}$ is unity.

Concerning the first of these points, activity coefficients of liquid components are variable, as demonstrated by coexisting immiscible liquids for which the thermodynamic activity of a given oxide component is the same in each liquid, but molar percents may be very different (Table 1). Indeed, if we consider only the terms in concentration in Eq. (3) and calculate $K_{M \text {-whit }}$ for each of our whitlockite saturated liquids: 


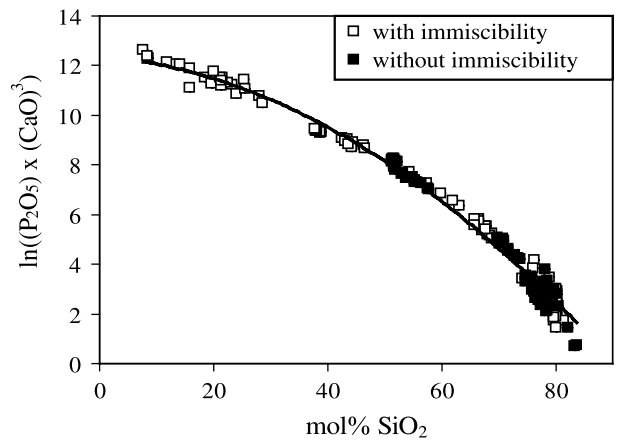

Fig. 8. Variation of $\ln \left(K_{M \text {-whit }}\right)$ for whitlockite saturated liquids at $1055^{\circ} \mathrm{C}$ (see text for details) as a function of $\mathrm{mol}^{\circ} \% \mathrm{SiO}_{2}$ assuming no effect of substitutions of $\mathrm{Mg}$ and $\mathrm{Fe}$ for $\mathrm{Ca}$ (see text for details). Error bars are typically smaller than the size of the symbols.

$K_{M \text {-whit }}=\left(M_{\mathrm{CaO}}^{\mathrm{liq}}\right)^{3} \times\left(M_{\mathrm{P}_{2} \mathrm{O}_{5}}^{\mathrm{liq}}\right)$,

where $M$ is the mole percent of the relevant oxide in the liquid (scale from 0 to 100 ), we find a variation in $K_{M \text {-whit }}$ of almost five orders of magnitude, in turn implying the same variability in the product of activity coefficients (cf. Eq. (3)). However, despite this wide range, $K_{M \text {-whit }}$ is found to be a systematic function of the $\mathrm{SiO}_{2}$ mole percent of the liquid (Fig. 8). Of particular note is the fact that data from systems showing immiscibility and those not showing immiscibility define the same trend. The scatter is somewhat greater at high $\mathrm{SiO}_{2}$ content, but remains of the same order of magnitude as uncertainties propagated from the electron-microprobe analyses of $\mathrm{CaO}$ and $\mathrm{P}_{2} \mathrm{O}_{5}$.

Concerning the second issue, a complete and rigorous assessment of the activity of $\mathrm{Ca}_{3}\left(\mathrm{PO}_{4}\right)_{2}$ in our experimental phosphates should take into account mixing of $\mathrm{Ca}, \mathrm{Mg}$ and $\mathrm{Fe}$ in the whitlockite structure. These cations will be concentrated on one or two of the five possible sites (Ca5 \pm Ca4; Calvo and Gopal, 1975; Nord, 1983; Belik et al., 2002 and references therein). Furthermore, a quantitative understanding of the energetics of mixing at each of the sites occupied by $\mathrm{Fe}$ and $\mathrm{Mg}$ is required. Calculations based on detailed consideration of site occupancy would therefore be relatively complex and necessarily qualitative given the available structural and thermodynamic data. On the other hand, we note that all the whitlockites in our experiments have approximately the same concentration of calcium, and that the correlation observed in Fig. 8 is well defined. In light of these latter observations we conclude that even though the activity of $\mathrm{Ca}_{3}\left(\mathrm{PO}_{4}\right)_{2}$ is clearly not unity for the phosphates in our experiments, to a first approximation a constant value may be assumed.

In any case, from a practical point of view the trend shown in Fig. 8 provides a simple and powerful way to describe the compositional characteristics of our whitlockite saturated silicate liquids at $1055^{\circ} \mathrm{C}$. This trend may be described by the equation:

$$
\begin{aligned}
\ln & {\left[\left(M_{\mathrm{CaO}}^{\mathrm{liq}}\right)^{3} \times\left(M_{\mathrm{P}_{2} \mathrm{O}_{5}}^{\mathrm{liq}}\right)\right] } \\
& =-0.0015\left(M_{\mathrm{SiO}_{2}}^{\mathrm{liq}}\right)^{2}-0.0052\left(M_{\mathrm{SiO}_{2}}^{\mathrm{liq}}\right)+12.147 .
\end{aligned}
$$

Mole percentages rather than mole fractions have been used here to underline the fact that although this equation is based upon the thermodynamic formalism presented above, it is not a rigorous thermodynamic expression. The simplicity of Eq. (5), which requires no knowledge of how liquid composition, liquid structure and activity coefficients of $\mathrm{CaO}$ and $\mathrm{P}_{2} \mathrm{O}_{5}$ are related, is somewhat surprising. Indeed, it may be argued that a trend is observed in Fig. 8 because all our experiments are multiply saturated in other mineral phases (e.g., systematic presence of plagioclase and a calcium-bearing pyroxene) which thus controls or at least limits the thermodynamic activities of silica and/ or lime in our liquids. Below we will therefore apply the formalism developed above to experimental data from the literature which are not necessarily multiply saturated, including extension to apatite saturated liquids.

\subsubsection{Extension to apatite}

Apatite rather than whitlockite is the most abundant phosphate in terrestrial rocks, thus it is of interest to assess to what extent the compositional controls on apatite saturation are the same as those observed for whitlockite (cf. Fig. 8). Although our experiments did not contain apatite, sufficient experimental data are available in the literature to extend our formalism, at least to the case of fluorapatite saturated liquids. In the case of apatite, the solubility product may be written:

$K_{\mathrm{Ca}_{5}\left(\mathrm{PO}_{4}\right)_{3}(\mathrm{~F}, \mathrm{Cl})}=\left(a_{\mathrm{CaO}}^{\mathrm{liq}}\right)^{5} \times\left(a_{\mathrm{P}_{2} \mathrm{O}_{5}}^{\mathrm{liq}}\right)^{1.5} \times\left(a_{\mathrm{F}, \mathrm{Cl}}^{\mathrm{liq}}\right) /\left(a_{\mathrm{Ca}_{5}\left(\mathrm{PO}_{4}\right)_{3}(\mathrm{~F}, \mathrm{Cl})}^{\text {apate }}\right)$.

Comparison of Eqs. (2) and (6) shows that apatite saturation differs from that of whitlockite because of the presence of volatiles in the former, and because the $\mathrm{Ca} / \mathrm{P}$ ratio of the crystal is different. Concerning the presence of volatiles, for the present purposes we will consider only experimental data in which liquids were saturated in fluorapatite by dissolution (Watson, 1979; Sha, 2000). In this case the activity of halogens in the liquid can be considered approximately constant, and thus should not affect the variation of the equilibrium constant as a function of melt composition. In an analogous way to $K_{M \text {-whit }}$ we define the parameter $K_{M \text {-apatite, }}$ expressed as:

$K_{M \text {-apatite }}=\left(M_{\mathrm{CaO}}^{\mathrm{liq}}\right)^{5} \times\left(M_{\mathrm{P}_{2} \mathrm{O}_{5}}^{\mathrm{liq}}\right)^{1.5}$.

The different stoichiometry of apatite and whitlockite (i.e., the different $\mathrm{Ca} / \mathrm{P}$ ) has the consequence that the absolute values of $K_{M \text {-apatite }}$ and $K_{M \text {-whit }}$ cannot be directly compared. One solution to this problem is to use a common basis for all liquid compositions irrespective of the crystalline phosphate in which they are saturated (i.e., consistent 
use of either $K_{M \text {-apatite }}$ or $K_{M \text {-whit }}$ for all liquids). In this way, it may be assessed whether liquid compositions saturated in these two different phosphates are comparable or not. For this comparison we require data for whitlockite and apatite saturated liquids at the same temperature. The literature data for fluorapatite saturated liquids which cover a wide range of $\mathrm{SiO}_{2}$ content (Watson, 1979) are generally at higher temperature than our experiments. On the other hand, the data of Watson (1979) for fluorapatite saturated liquids at $1200{ }^{\circ} \mathrm{C}$ may be compared with those of Sha (2000) for whitlockite saturated liquids at the same temperature (Fig. 9). This comparison of values of $K_{M \text {-apa- }}$ tite shows that a single trend is apparent as a function of $\mathrm{SiO}_{2}$ content which, furthermore, shares many features of the trend defined by our data at lower temperature, as discussed further below.

\subsubsection{The effect of temperature}

In addition to the importance of liquid composition, the model of Harrison and Watson (1984) implies that temperature also plays a role on apatite saturation, increasing temperature leading to higher levels of $\mathrm{P}_{2} \mathrm{O}_{5}$ in the liquid at $\mathrm{P}$ saturation. Although the temperature range of our experiments is not sufficient to observe this effect (Fig. 6a), our data combined with those of Watson (1979), Pichavant et al. (1992) and Sha (2000) cover temperatures from 777 to $1400{ }^{\circ} \mathrm{C}$. When $\ln \left(K_{M \text {-apatite }}\right)$ is plotted as a function of molar percent of $\mathrm{SiO}_{2}$, liquids from experiments at different temperatures (e.g., isothermal sections at 1055, 1200, 1300 and $1400{ }^{\circ} \mathrm{C}$ ) clearly define a series of parallel trends (Fig. 10). Indeed, we find that when $K_{M \text {-apatite }}$ is divided by temperature, all experimental liquids considered define a single trend (Fig. 11). Although this way of incorporating temperature is purely empirical, it provides a simple way to express the compositional and temperature effects that characterise liquids saturated in fluorapatite and/or whitlockite and which may thus be used as the basis for a comprehensive predictive model, detailed below.

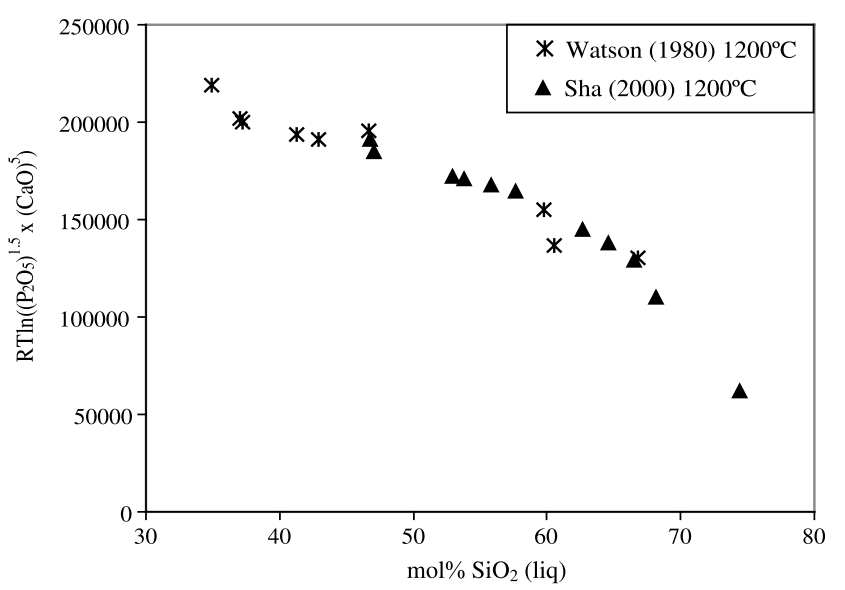

Fig. 9. Variation of $\ln \left(K_{M \text {-apatite }}\right)$ as a function of $\mathrm{mol} \% \mathrm{SiO}_{2}$ for whitlockite and fluorapatite saturated liquids at $1200^{\circ} \mathrm{C}$ (see text for details of this comparison). Data sources as indicated in the key.

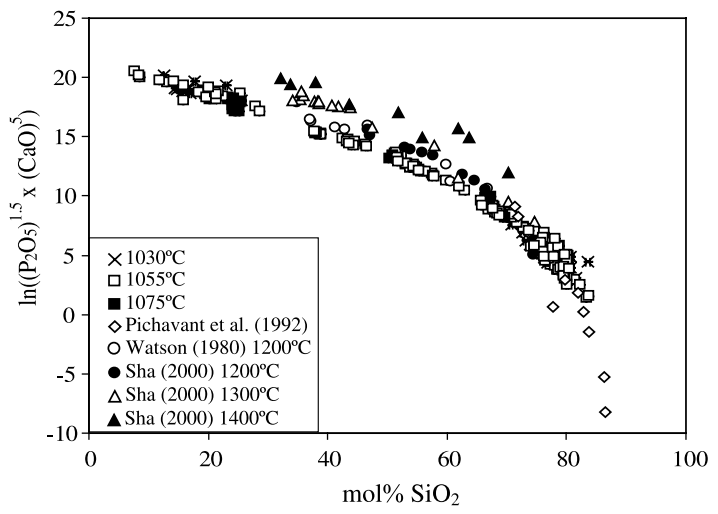

Fig. 10. Variation of $\ln \left(K_{M \text {-apatite }}\right)$ as a function of $\mathrm{mol}^{2} \% \mathrm{SiO}_{2}$ (see text for definition of $K_{M \text {-apatite }}$. Data sources as indicated in the key. Error bars are typically smaller than the size of the symbols.

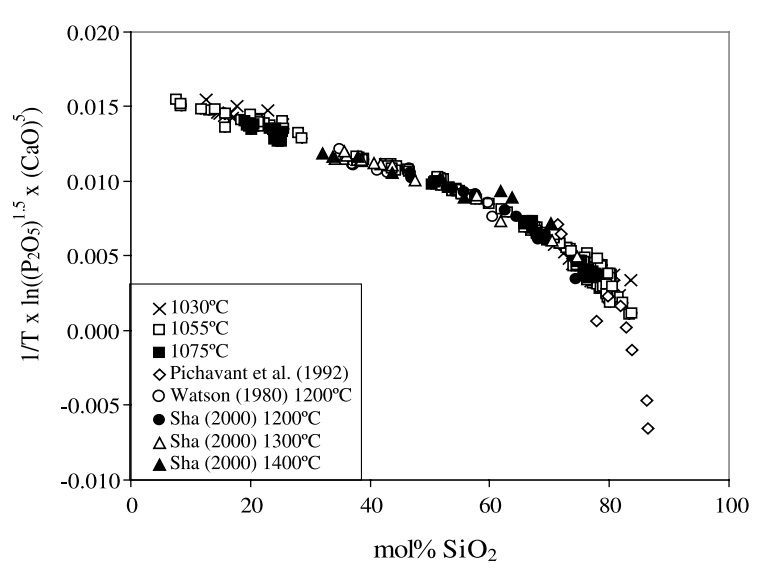

Fig. 11. Variation of $\ln \left(K_{M \text {-apatite }}\right) / T$ as a function of $m o l \% \mathrm{SiO}_{2}$. Data sources as indicated in the key. Error bars are typically smaller than the size of the symbols.

\subsection{The case of peraluminous liquids}

One notable feature of Figs. 10 and 11 is that the peraluminous liquids of Pichavant et al. (1992) follow exactly the same trend as the subaluminous and peralkaline liquids studied by other authors. This result therefore implies that the discrepancy between the $\mathrm{P}_{2} \mathrm{O}_{5}$ content at apatite saturation measured by Pichavant et al. (1992) and that predicted by the model of Harrison and Watson (1984) is an indirect effect of $\mathrm{CaO}$ content (which is not accounted for in the model of Harrison and Watson, 1984) rather than a direct effect of the peraluminous nature of the liquids. A similar conclusion regarding the importance of $\mathrm{CaO}$ was proposed by Bea et al. (1992) based upon consideration of data from natural peraluminous granitic magmas.

\subsection{A predictive model for phosphate saturation}

The data shown in Fig. 11 represent liquids saturated in either fluorapatite, $\alpha$-whitlockite (data of Sha (2000) at $1400{ }^{\circ} \mathrm{C}$ ) or $\beta$-whitlockite. These liquids are highly variable in composition and are not systematically saturated in 
other phases which may buffer the activities of certain melt components. For example, the liquids of Sha (2000) are saturated in phosphate alone, while in the study of Pichavant et al. (1992) corundum and andalusite are reported as accompanying phases. We therefore conclude that the correlation observed in Fig. 11 may be used as the basis for a comprehensive model for calculation of the saturation of fluorapatite or whitlockite from silicate magmas. Indeed, the fact that data for different phosphate minerals are not distinguished in Fig. 11 implies that a given silicate liquid will either contain no phosphate, or will be saturated in one of apatite or whitlockite, crystallisation of apatite presumably occurring in the presence of sufficient $\mathrm{Cl}$ or $\mathrm{F}$, and whitlockite in volatile-free systems. Whether or not this correlation is also valid for hydroxyapatite saturated liquids remains to be established.

Quantitatively, the data of Fig. 11 may be described as a function of silica content by the equation:

$$
\begin{aligned}
\frac{1}{T} & \ln \left[\left(M_{\mathrm{CaO}}^{\mathrm{Liq}}\right)^{5} \times\left(M_{\mathrm{P}_{2} \mathrm{O}_{5}}^{\mathrm{liq}}\right)^{1.5}\right] \\
& =\left\{\frac{-1.2868}{139.00-M_{\mathrm{SiO}_{2}}^{\mathrm{liq}}}+0.0247\right\} .
\end{aligned}
$$

The quadratic form used in Eq. (5) has been avoided here in order to eliminate high-order terms in silica concentration of the liquid. Eq. (8) may then be rearranged to define the $\mathrm{mol} \% \mathrm{P}_{2} \mathrm{O}_{5}$ of a liquid saturated in apatite/whitlockite $\left(M_{\mathrm{P}_{2} \mathrm{O}_{5}}^{\text {liq-at }}\right)$, as a function of temperature and the $\mathrm{mol} \% \mathrm{SiO}_{2}$ and $\mathrm{CaO}$ of the liquid:

$$
\begin{aligned}
M_{\mathrm{P}_{2} \mathrm{O}_{5}}^{\text {liq-sat }}= & \exp \left[\frac { 2 } { 3 } \left(T\left\{\frac{-1.2868}{139.00-M_{\mathrm{SiO}_{2}}^{\mathrm{liq}}}+0.0247\right\}\right.\right. \\
& \left.\left.-5 \ln \left(M_{\mathrm{CaO}}^{\mathrm{liq}}\right)\right)\right] .
\end{aligned}
$$

\subsection{Application to natural systems}

Despite the relative simplicity of Eq. (9), one shortcoming of the model presented above is that concentrations are expressed in $\mathrm{mol} \%$ rather than $\mathrm{wt} \%$. Not only does this render interpretation of values less intuitive than if they were in $\mathrm{wt} \%$, but this also has the drawback that unless the whole liquid composition is known the model cannot be used. This could be problematic in geological environments (i.e., layered intrusions) where the liquid is no longer present. One way around this problem is to define factors which allow conversion from $\mathrm{wt} \%$ to $\mathrm{mol} \%$ and vice versa. This is not as trivial as may seem at first glance, because the $\mathrm{mol} \%$ of a given oxide will depend on the fractions and molar weights of the other components. However, when the $\mathrm{mol} \%$ and $\mathrm{wt} \%$ of a given oxide are compared for our liquids with $\mathrm{SiO}_{2}$ content in the range $30-75 \mathrm{wt} \%$ (i.e., those most representative of natural compositions), the data define excellent linear correlations which pass through the
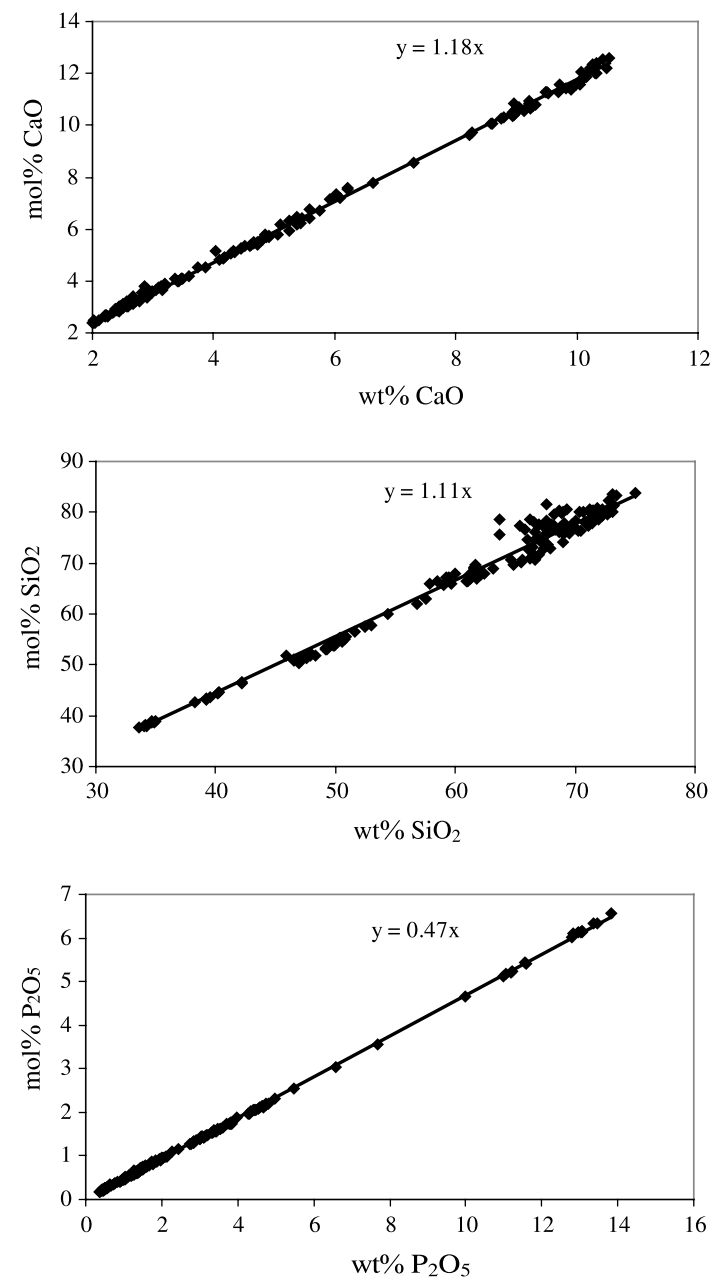

Fig. 12. Relations between weight percent and mole percent for components $\mathrm{CaO}, \mathrm{SiO}_{2}$ and $\mathrm{P}_{2} \mathrm{O}_{5}$ in typical magmatic compositions. Equations are given in the text.

origin (Fig. 12). Based upon these correlations we determine that for geologically relevant liquids: $\mathrm{mol} \%$ $\mathrm{SiO}_{2}=1.11 * \mathrm{wt}^{2} \% \mathrm{SiO}_{2} ; \mathrm{mol} \% \mathrm{CaO}=1.18 * \mathrm{wt} \% \mathrm{CaO} ;$ and $\mathrm{mol} \% \mathrm{P}_{2} \mathrm{O}_{5}=0.47 * \mathrm{wt} \% \mathrm{P}_{2} \mathrm{O}_{5}$. Using these correction factors, Eq. (9) can be used even when only wt $\%$ analyses are known or can be assumed, although we stress that molar values should be used wherever possible.

This equation may be used in several ways, either to assess whether a given liquid is saturated in phosphate or not, or rearranged to constrain the composition and/or temperature of a system which is known to be saturated in either apatite or whitlockite. From a more general perspective, it may also be used to illustrate the importance of each parameter $\left(\mathrm{T}, \mathrm{CaO}\right.$, and $\left.\mathrm{SiO}_{2}\right)$ on $\mathrm{wt} \% \mathrm{P}_{2} \mathrm{O}_{5}$ required for phosphate saturation. For example, at fixed $\mathrm{CaO}$ content of the liquid Eq. (9) may be used to show that the effect of changing $\mathrm{SiO}_{2}$ content dominates the effect of changing temperature (Fig. 13a). Furthermore, the effect of temperature is particularly small for $\mathrm{SiO}_{2}$ content greater than $55 \mathrm{~mol} \%$ (Fig. 13a). At fixed temperature (of $1050{ }^{\circ} \mathrm{C}$ ), it is predicted that the effect of $\mathrm{CaO}$ is negligible at high $\mathrm{SiO}_{2}$ content, but may become extremely important at lower 

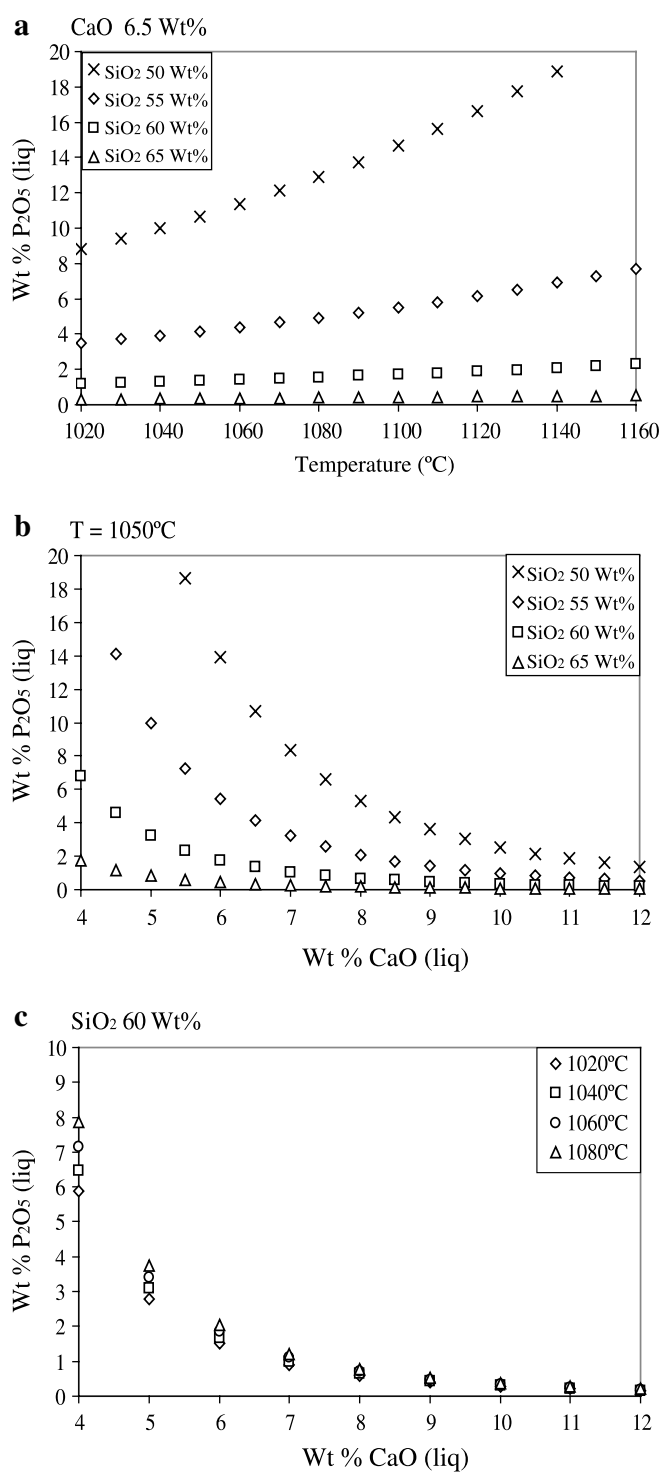

Fig. 13. Calculated values of $w t \% \mathrm{P}_{2} \mathrm{O}_{5}$ required for phosphate saturation as a function of $\mathrm{wt} \% \mathrm{SiO}_{2}, \mathrm{CaO}$ and temperature.

$\mathrm{SiO}_{2}$ content (Fig. 13b). In other words, in granitic systems the influence of $\mathrm{CaO}$ concentration of the liquid on $\mathrm{wt} \%$ $\mathrm{P}_{2} \mathrm{O}_{5}$ required for phosphate saturation will be minor, but this will not be true in low- $\mathrm{SiO}_{2}$ basaltic systems. Finally, at fixed $\mathrm{SiO}_{2}$ content, it may be appreciated that the effect of $\mathrm{CaO}$ dominates that of temperature, although the effect of temperature is more marked at low $\mathrm{CaO}$ content (Fig. 13c). Despite the fact that the effects of changing $\mathrm{SiO}_{2}$ and $\mathrm{CaO}$ concentrations in the liquid dominate those of changing temperature, it is clear that there is a complex interdependence of temperature, $\mathrm{SiO}_{2}$ and $\mathrm{CaO}$ concentration on $\mathrm{wt} \% \mathrm{P}_{2} \mathrm{O}_{5}$ required for phosphate saturation and that Eq. (9) should be used for any quantitative application.

\subsubsection{Illustrating saturation as a function of magmatic differentiation}

Although Eq. (9) may be used to calculate how temperature and melt composition affect $\mathrm{wt} \% \mathrm{P}_{2} \mathrm{O}_{5}$ required for phosphate saturation, as illustrated in Fig. 13, it is not immediately obvious how this parameter will vary along the liquid line of descent of a real magmatic system. Indeed, how $\mathrm{wt}^{\mathrm{t}} \% \mathrm{P}_{2} \mathrm{O}_{5}$ required for phosphate saturation varies in response to the changes in major element composition of the liquid caused by fractional crystallisation will obviously be a key factor which will determine at which point along the liquid line of descent a phosphate will appear. The other key factor is the evolution of the phosphorus content of the liquid as differentiation progresses, saturation occurring when the $\mathrm{P}_{2} \mathrm{O}_{5}$ content of the liquid becomes greater than the $\mathrm{wt} \% \mathrm{P}_{2} \mathrm{O}_{5}$ required for phosphate saturation. This is illustrated below for the case of differentiation of a ferrobasaltic magma along the FMQ buffer using the experimentally determined liquids described by Toplis and Carroll (1995). The initial liquid used in that study has the composition of a dyke found close to the Skaergaard intrusion and which has been proposed as a possible parental magma of that intrusion (Brooks and Nielsen, 1978). A detailed account of the phase relations and liquid compositions can be found in Toplis and Carroll (1995) although the salient features are summarised below.

For each individual liquid of Toplis and Carroll (1995) the value of $\mathrm{wt} \% \mathrm{P}_{2} \mathrm{O}_{5}$ required for phosphate saturation has been calculated and is reported as a function of temperature in Fig. 14. For the different liquid compositions produced during cooling, the calculated variation of $\mathrm{wt} \% \mathrm{P}_{2} \mathrm{O}_{5}$ required for phosphate saturation defines three distinct segments. The one at highest temperature is characterised by decreasing $\mathrm{wt}^{\%} \% \mathrm{P}_{2} \mathrm{O}_{5}$ required for phosphate saturation with falling temperature. In this range, olivine and plagioclase are the only liquidus phases and the liquid has approximately constant $\mathrm{CaO}$ and $\mathrm{SiO}_{2}$ content (Toplis and Carroll, 1995). The calculated variation in $\mathrm{wt}_{\mathrm{t}} \% \mathrm{P}_{2} \mathrm{O}_{5}$

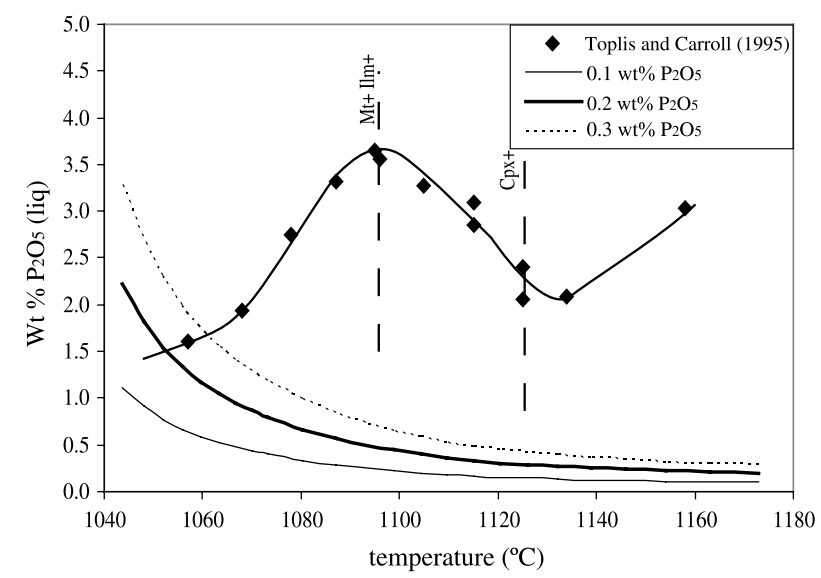

Fig. 14. Values of $\mathrm{wt} \% \mathrm{P}_{2} \mathrm{O}_{5}$ required to saturate experimental liquids of Toplis and Carroll (1995) in a crystalline phosphate calculated using Eq. (9) (diamonds). Solid and dashed curves represent $w t \% \mathrm{P}_{2} \mathrm{O}_{5}$ of the liquid as a function of temperature assuming that the initial liquids contained $0.1,0.2$ and $0.3 \mathrm{wt} \% \mathrm{P}_{2} \mathrm{O}_{5}$ (as labelled). Intersection of the curves and diamonds may be used to predict phosphate saturation as detailed in the text. Phases: $\mathrm{Cpx}$, clinopyroxene; $\mathrm{Mt}$, magnetite-ulvöspinel solid solution; Ilm, ilmenite-haematite solid solution. 
required for phosphate saturation can thus be attributed to the change in temperature. A marked change in the behaviour of $\mathrm{wt} \% \mathrm{P}_{2} \mathrm{O}_{5}$ required for phosphate saturation occurs at $\sim 1130{ }^{\circ} \mathrm{C}$ (Fig. 14), corresponding to the appearance of clinopyroxene on the liquidus (Toplis and Carroll, 1995). In the temperature range $1130-1100{ }^{\circ} \mathrm{C}$, the $\mathrm{SiO}_{2}$ content of the liquid is approximately constant, but the $\mathrm{CaO}$ content of the liquid is decreasing. Therefore, the marked increase in $\mathrm{wt}_{0} \% \mathrm{P}_{2} \mathrm{O}_{5}$ required for phosphate saturation can be attributed to the falling $\mathrm{CaO}$ content of the liquid, which dominates the effect of falling temperature. The second major change in the behaviour of $\mathrm{wt} \% \mathrm{P}_{2} \mathrm{O}_{5}$ required for phosphate saturation occurs at $\sim 1100{ }^{\circ} \mathrm{C}$ (Fig. 14) when magnetite appears on the liquidus. For temperatures below $1100{ }^{\circ} \mathrm{C}$, the $\mathrm{CaO}$ content of the liquid continues to decrease, but the $\mathrm{SiO}_{2}$ content of the liquid increases with falling temperature. The drop in wt $\% \mathrm{P}_{2} \mathrm{O}_{5}$ required for phosphate saturation with decreasing temperature can therefore be attributed to the increase in $\mathrm{SiO}_{2}$ content, an effect which dominates those of $\mathrm{CaO}$ content and temperature which are both acting to increase wt $\% \mathrm{P}_{2} \mathrm{O}_{5}$ required for phosphate saturation.

These values of $\mathrm{wt} \% \mathrm{P}_{2} \mathrm{O}_{5}$ required for phosphate saturation may then be compared with the theoretical evolution of $\mathrm{P}_{2} \mathrm{O}_{5}$ in the liquid to assess at what point phosphate saturation would occur. For example, assuming that phosphorous is perfectly incompatible and using the variation of percentage crystallisation as a function of temperature determined by Toplis and Carroll (1995), one predicts that if the liquid at $1170{ }^{\circ} \mathrm{C}$ contained $0.2 \mathrm{wt} \% \mathrm{P}_{2} \mathrm{O}_{5}$, then phosphate saturation should occur at $1055^{\circ} \mathrm{C}$ when the liquid contains $1.5 \mathrm{wt} \% \mathrm{P}_{2} \mathrm{O}_{5}$ (Fig. 14). A concentration of $\mathrm{P}_{2} \mathrm{O}_{5}$ in the initial liquid of $0.1 \mathrm{wt} \% \mathrm{P}_{2} \mathrm{O}_{5}$ leads to slightly lower saturation temperatures and a concentration of $\mathrm{P}_{2} \mathrm{O}_{5}$ in the liquid at saturation of approximately $1 \mathrm{wt} \%$ (Fig. 14). These ranges of temperature and $\mathrm{P}_{2} \mathrm{O}_{5}$ content are perfectly consistent with independent estimates proposed for the Skaergaard intrusion (Wager, 1960; McBirney and Naslund, 1990), providing evidence for the validity of the model.

\subsubsection{The petrogenesis of rocks dominated by association of apatite and $\mathrm{Fe}-\mathrm{Ti}$ oxide}

Rocks dominated by the presence of apatite and $\mathrm{Fe}-\mathrm{Ti}$ oxide may be found in diverse magmatic environments, including mafic intrusions, rocks associated with anorthosites, and ophiolites (e.g., Wager and Brown, 1967; Philpotts, 1967; Dymek and Owens, 2001; Mitsis and Economou-Eliopoulos, 2001). The petrogenesis of these rock types, in particular that of nelsonites, is the subject of debate. The principal proposals are: (1) that nelsonites represent the crystallisation products of an immiscible $\mathrm{Fe}-\mathrm{Ti}$ oxide liquid which separated from a silicate magma (e.g., Philpotts, 1967; Ripley et al., 1998), an immiscibility which may be linked to magma mixing (e.g., Clark and Kontak, 2004); (2) that apatite and oxide represent cumulates from an evolved silicate magma (e.g., Emslie, 1975; Dymek and Owens, 2001; Barnes et al., 2004).
Even though the experimental results presented here show immiscibility, the bulk $\mathrm{P}_{2} \mathrm{O}_{5}$ contents of our starting compositions are unrealistically high for natural ferrobasaltic liquids and any direct application of our results to the question of nelsonite petrogenesis is probably unfounded. On the other hand, the calculated variation of $\mathrm{wt}^{0} \% \mathrm{P}_{2} \mathrm{O}_{5}$ required for phosphate saturation during ferrobasaltic differentiation (Fig. 14) may be used to assess whether or not extremely high levels of $\mathrm{P}$ and $\mathrm{Fe}$ may be reached in the liquid, without the need to postulate liquid immiscibility. For example, Fig. 14 shows that during cotectic crystallisation of olivine, plagioclase and clinopyroxenes, $\mathrm{wt} \% \mathrm{P}_{2} \mathrm{O}_{5}$ required for phosphate saturation increases sharply, a trend which is only reversed by the appearance of magnetite. As discussed above this is the consequence of decreasing $\mathrm{CaO}$ content of the liquid during crystallisation of olivine gabbro (Shi and Libourel, 1991; Toplis and Carroll, 1995). Thus, if the appearance of magnetite on the liquidus is displaced to lower temperature (i.e., the middle segment in Fig. 14 extends to lower temperature, and thus higher values of $\mathrm{wt} \%$ $\mathrm{P}_{2} \mathrm{O}_{5}$ required for phosphate saturation), the saturation temperature of phosphate will also be lowered and the concentrations of $\mathrm{P}_{2} \mathrm{O}_{5}$ in the liquid will be significantly higher when a phosphate does finally appear. We therefore conclude that retarding magnetite saturation will indeed tend to retard phosphate saturation, but not because ferric iron stabilises $\mathrm{P}$ in the liquid as originally postulated, but rather as a consequence of the variations of $\mathrm{CaO}$ and $\mathrm{SiO}_{2}$ content of the liquid as a function of magmatic differentiation.

The fact that the presence of $\mathrm{P}$ in ferrobasaltic liquids retards magnetite crystallisation (Toplis et al., 1994a) therefore provides a chemical mechanism to enrich magmatic liquids to high concentrations of both $\mathrm{Fe}$ and $\mathrm{P}$. However, the extent to which this mechanism is relevant to the petrogenesis of nelsonites remains to be demonstrated. For example, nelsonites typically contain less than $10 \%$ silicate minerals, although more than $10 \%$ of such phases were present in all of our experiments. We conclude that further experimental work and petrographic study of natural nelsonites is required to understand the physical and chemical mechanisms which lead to the formation of these enigmatic rocks.

\section{Concluding remarks}

In the light of our experimental results, and those in the literature, we conclude that it is $\mathrm{SiO}_{2}$ and $\mathrm{CaO}$ concentrations of the liquid that dominate phosphate saturation in magmatic systems, any effect of iron and/or oxidation state being of secondary importance. Based upon these results we propose an equation which may be used to predict the $\mathrm{P}_{2} \mathrm{O}_{5}$ concentration of silicate liquids saturated either in whitlockite or apatite as a function of melt chemistry and temperature. Of particular note is the fact that this equation is valid over extremely wide ranges of liquid composition (e.g., $\mathrm{SiO}_{2}$ content from 10 to $75 \mathrm{~mol} \%$ ), not only for peralkaline and subaluminous compositions, but also peraluminous liquids. 


\section{Acknowledgments}

This work was supported financially by a Canada Research Chair and Natural Sciences and Engineering Council Discovery Grants to S.J.B. and a CNRS grant (Intérieur de la Terre) to M.T. The referees are thanked for giving up their valuable time to review this manuscript. This work has benefited from the help of numerous people and N.T. would like to acknowledge the support of Richard LeBreton, Fabien Solgadi, Tafadzwa Gomwe, Bélinda Godel, Fabien Palhol, Laurent Tissandier, and the microprobe technicians of the Service Commun de Micro-Analyse at the Université Henri Poincaré in Nancy.

\section{Associate editor: Edward M. Ripley}

\section{References}

Barnes, S.J., Maier, W.D., Ashwal, L.D., 2004. Platinum-group element distribution in the Main Zone and in the Upper Zone of the Northern Limb of the Bushveld Complex. Chem. Geol. 208, 293-317.

Bea, F., Fershtater, G., Corretge, L.G., 1992. The geochemistry of phosphorus in granite rocks and the effect of aluminium. Lithos 29, 43-56.

Belik, A.A., Izumi, F., Ikeda, T., Okui, M., Malakho, A.P., Morozov, V.A., Lazoryak, B.I., 2002. Whitlockite-related phosphates $\mathrm{Sr}_{9} \mathrm{~A}\left(\mathrm{PO}_{4}\right)_{7}$ $(\mathrm{A}=\mathrm{Sc}, \mathrm{Cr}, \mathrm{Fe}, \mathrm{Ga}$, and $\mathrm{In})$ : structure refinement of $\mathrm{Sr}_{9} \mathrm{In}\left(\mathrm{PO}_{4}\right)_{7}$ with synchrotron X-ray powder diffraction data. J. Solid State Chem. 168, 237-244.

Brooks, C.K., Nielsen, T.F.D., 1978. Early stages in the differentiation of the Skaergaard magma as revealed by closely related suite of dye rocks. Lithos 11, 1-14.

Brooks, C.K., Larsen, L.M., Nielsen, T.F.D., 1991. Importance of ironrich tholeiitic magmas at divergent plate margins: a reappraisal. Geology 19, 269-272.

Calvo, C., Gopal, R., 1975. The crystal structure of whitlockite from the Palermo quarry. Am. Mineral. 60, 120-133.

Cimon, J., 1998. L'unité à apatite de rivière des rapides, Complexe de Sept îles. Localisation stratigraphique et facteurs à l'origine de sa formation. Ministère des ressources naturelles, Québec 97, 1-32.

Clark, A.H., Kontak, D.J., 2004. Fe-Ti-P oxide melts generated through magma mixing in the Antuata Subvolcanic Center, Peru: implications for the origin of nelsonite and iron-oxide dominated hydrothermal deposits. Econ. Geol. 99, 377-395.

Davies, G., Cawthorn, R.G., 1984. Mineralogical data on a multiple intrusion in the Rustenburg Layered Suite of the Bushveld Complex. Mineral. Mag. 48, 469-480.

Deines, P., Hafzinger, R.H., Ulmer, G.C., Woermann, E., 1974. Temperature-oxygen fugacity tables for selected gas mixtures in the system $\mathrm{C}-$ $\mathrm{H}-\mathrm{O}$ at one atmosphere total pressure. In: Bulletin of the Earth and Mineral Sciences Experiment Station, vol. 88. Pennsylvania State University, Pennsylvania, p. 128.

Delaney, J.S., O'Neill, C., Prinz, M., 1984. Phosphate minerals in eucrites. Lunar Planet. Sci. XV, 208-209 (abstr.).

Dymek, R.F., Owens, B.E., 2001. Petrogenesis of apatite-rich rocks (nelsonites and oxide-apatite gabbros) associated with massif anorthosites. Econ. Geol. 96, 797-815.

Emslie, R.F., 1975. Major rock units of the Morin Complex, southwestern Quebec. Geol. Survey of Canada Paper 74-48, 37pp.

Gan, H., Hess, P.C., 1992. Phosphate speciation in potassium aluminosilicate glasses. Am. Mineral. 77, 495-506.

Ghiorso, M.S., Carmichael, I.S.E., Rivers, M.L., Sack, R.O., 1983. The Gibbs free energy of mixing of natural silicate liquids; an expanded regular solution approximation for the calculation of magmatic intensive variables. Contrib. Mineral. Petrol. 84, 107-145.
Griffen, W.L., Amli, R., Heier, K.S., 1972. Whitlockite and apatite from lunar rock 14310 and from Ödegärden, Norway. Earth Planet. Sci. Lett. 15, 53-58.

Gwinn, R., Hess, P.C., 1993. The role of phosphorus in rhyolitic liquids as determined from the homogenous iron redox equilibrium. Contrib. Mineral. Petrol. 106, 129-141.

Harney, D.M.W., Von Gruenewaldt, G., 1995. Ore-forming processes in the upper part of the Bushveld complex, South Africa. J. Afr. Earth Sci. 20, 77-89.

Harrison, T.M., Watson, E.B., 1984. The behavior of apatite during crustal anatexis: equilibrium and kinetic considerations. Geochim. Cosmochim. Acta 48, 1467-1477.

Huminicki, D.M.C., Hawthorne, F.C., 2002. The crystal chemistry of phosphate mineral. In: Kohn, M.J., Rakovan, J., Hughes, J.M. (Eds.), Phosphates-Geochemical, Geobiological and Materials Importance. Mineral. Soc. Am. Reviews in Mineralogy, vol. 48, pp. 123-235.

Kilinc, A., Carmichael, I.S.E., Rivers, M.L., Sack, R.O., 1983. The ferricferrous ratio of natural silicate liquids equilibrated in air. Contrib. Mineral. Petrol. 83, 136-140.

Kushiro, I., 1975. On the nature of silicate melt and its significance in magma genesis: regularities in the shift of the liquidus boundaries involving olivine, pyroxene, and silica minerals. Am. J. Sci. 275, 411-431.

Libourel, G., 1999. Systematics of calcium partitioning between olivine and silicate melt: implications for melt structure and calcium content of magmatic olivines. Contrib. Mineral. Petrol. 136, 63-80.

Linnen, R.L., Keppler, H., 1997. Columbite solubility in granitic melts: consequences for the enrichment and fractionation of $\mathrm{Nb}$ and $\mathrm{Ta}$ in the Earth's crust. Contrib. Mineral. Petrol. 128, 213-227.

Linnen, R.L., Keppler, H., 2002. Melt composition control on $\mathrm{Zr} / \mathrm{Hf}$ fractionation in magmatic processes. Geochim. Cosmochim. Acta 66, 3293-3301.

Lundberg, L.L., Crozaz, G., McKay, G., Zinner, E., 1988. Rare earth element carriers in the Shergotty meteorite and implications for its chronology. Geochim. Cosmochim. Acta 52, 2147-2163.

McBirney, A.R., Naslund, H.R., 1990. The differentiation of the Skaergaard intrusion. A discussion of R. H. Hunter and R. S. J. Sparks (Contrib. Mineral. Petrol. 95, 451-461). Contrib. Mineral. Petrol. 104, 235-240.

Mitsis, I., Economou-Eliopoulos, M., 2001. Occurrence of apatite associated with magnetite in an ophiolite complex. Am. Mineral. 86, 1143-1150.

Mysen, B.O., 1992. Iron and phosphorus in calcium silicate quenched melts. Chem. Geol. 98, 175-202.

Nabil, H., 2003. Genèse des dépôts de Fe-Ti-P associés aux intrusions litées (exemples: intrusion mafique de Sept-Îles, au Québec; Complexe de Duluth aux États-Unis). Ph.D. thesis, Université du Québec à Chicoutimi.

Naslund, H.R., 1983. The effect of oxygen fugacity on liquid immiscibility in iron-bearing silicate melts. Am. J. Sci. 283, 1034-1059.

Nord, A.G., 1983. Incorporation of divalent metals in whitlockite related beta-Ca ${ }_{3}\left(\mathrm{PO}_{4}\right)_{2}$. Neues Jb. Miner. Monat. 11, 489-497.

Philpotts, A.R., 1967. Origin of certain iron-titanium oxide and apatite rocks. Econ. Geol. 62, 303-315.

Pichavant, M., Montel, J.M., Richard, L.R., 1992. Apatite solubility in peraluminous liquids: experimental data and an extension of the Harrison-Watson model. Geochim. Cosmochim. Acta 56, 3855-3861.

Ripley, E.M., Severson, M.J., Hauck, S.A., 1998. Evidence for sulphide and $\mathrm{Fe}-\mathrm{Ti}$-P-rich liquid immiscibility in the Duluth Complex, Minnesota. Econ. Geol. 93, 1052-1062.

Ryerson, F.J., Hess, P.C., 1978. Implications of liquid-liquid distribution coefficients to mineral-liquid partitioning. Geochim. Cosmochim. Acta 42, 921-932.

Schaller, T., Rong, C., Toplis, M.J., Cho, H., 1999. TRAPDOR NMR investigations of phosphorus-bearing aluminosilicate glasses. J. NonCryst. Solids 248, 19-27.

Sha, L., 2000. Whitlockite solubility in silicate melts: some insights into lunar and planetary evolution. Geochim. Cosmochim. Acta 64, 32173236. 
Shi, P., Libourel, G., 1991. The effects of FeO on the system CMAS at low pressure and implications for basaltic differentiation processes. Contrib. Mineral. Petrol. 108, 129-145.

Toplis, M.J., Carroll, M.R., 1995. An experimental study of the influence of oxygen fugacity on $\mathrm{Fe}-\mathrm{Ti}$ oxide stability, phase relations, and mineralmelt equilibria in ferro-basaltic systems. J. Petrol. 36, 1137-1170.

Toplis, M.J., Schaller, T., 1998. A ${ }^{31}$ P MAS NMR study of glasses in the system $x \mathrm{Na}_{2} \mathrm{O}-(1-x) \mathrm{Al}_{2} \mathrm{O}_{3}-2 \mathrm{SiO}_{2}-y \mathrm{P}_{2} \mathrm{O}_{5}$. J. Non-Cryst. Solids 224, $57-68$.

Toplis, M.J., Libourel, G., Carroll, M.R., 1994a. The role of phosphorus in crystallisation processes of basalt: an experimental study. Geochim. Cosmochim. Acta 58, 797-810.

Toplis, M.J., Dingwell, D.B., Libourel, G., 1994b. The effect of phosphorus on the iron redox ratio, viscosity, and density of an evolved ferrobasalt. Contrib. Mineral. Petrol. 117, 293-304.

Vermark, C.F., Von Gruenewaldt, G., 1986. Introduction to the Bushveld Complex. In: Mineral Deposits of Southern Africa, vol. 2, pp. 1021-1029.
Visser, W., Koster van Groos, A.F., 1979. Effects of $\mathrm{P}_{2} \mathrm{O}_{5}$ and $\mathrm{TiO}_{2}$ on liquid-liquid equilibria in the system $\mathrm{K}_{2} \mathrm{O}-\mathrm{FeO}-\mathrm{Al}_{2} \mathrm{O}_{3}-\mathrm{SiO}_{2} . A m . J$. Sci. 279, 970-988.

Von Gruenewaldt, G., 1993. Ilmenite-apatite enrichments in the upper zone of the Bushveld complex: a major titanium-rock phosphate resource. Int. Geol. Rev. 35, 987-1000.

Wager, L.R., 1960. The major element variation of the layered series of the Skaergaard intrusion and a re-estimation of the average composition of the hidden layered series and of the successive residual magmas. $J$. Petrol. 1, 364-398.

Wager, L.R., Brown, G.M., 1967. Layered Igneous Rocks. Oliver and Boyd, Edinburgh.

Watson, E.B., 1979. Apatite saturation in basic to intermediate magmas. Geophys. Res. Lett. 6, 937-940.

Wolf, M.B., London, D., 1994. Apatite dissolution into peraluminous haplogranitic melts: an experimental study of solubilities and mechanisms. Geochim. Cosmochim. Acta 58, 4127-4145. 